\title{
Data report: permeabilities of sediments from the Kumano Basin transect off Kii Peninsula, Japan'
}

\author{
Katherine Rowe, ${ }^{2}$ Elizabeth Screaton, ${ }^{2}$ Junhua Guo, ${ }^{3}$ and Michael B. Underwood ${ }^{3}$
}

\begin{abstract}
Chapter contents

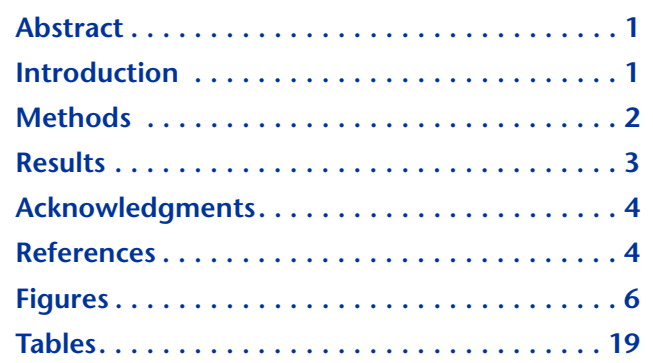

${ }^{1}$ Rowe, K., Screaton, E., Guo, J., and Underwood, M.B., 2011. Data report: permeabilities of sediments from the Kumano Basin transect off Kii Peninsula, Japan. In Kinoshita, M., Tobin, H., Ashi, J., Kimura, G., Lallemant, S., Screaton, E.J., Curewitz, D., Masago, H., Moe, K.T., and the Expedition 314/315/316 Scientists, Proc. IODP, 314/315/316: Washington, DC (Integrated Ocean Drilling Program Management International, Inc.). doi:10.2204/iodp.proc.314315316.211.2011

2Department of Geological Sciences, University of Florida, Gainesville FL 32661, USA.

Correspondence author: screaton@ufl.edu

${ }^{3}$ Department of Geological Sciences, University of Missouri, Columbia MO 65211, USA.

\begin{abstract}
Permeability tests were conducted on core samples from Integrated Ocean Drilling Program Expedition 315 Site C0001 and Expedition 316 Sites C0004, C0006, C0007, and C0008. Samples from Sites C0001, C0004, and C0008 represent sediments from the megasplay fault region from the Kumano Basin transect off Kii Peninsula, Japan, whereas samples from Sites C0006 and C0007 represent sediments from the frontal thrust region. Measured vertical permeabilities vary from $\sim 2.51 \times 10^{-19} \mathrm{~m}^{2}$ to $\sim 5.26 \times$ $10^{-16} \mathrm{~m}^{2}$, for a porosity range of $0.28-0.56$. Environmental scanning electron microscopy was used to evaluate the sediment fabric of samples collected adjacent to whole-round samples.
\end{abstract}

\section{Introduction}

Integrated Ocean Drilling Program (IODP) Expeditions 315 and 316 are part of Stage 1 of a multistage, multiexpedition effort known as the Nankai Trough Seismogenic Zone Experiment (NanTroSEIZE). NanTroSEIZE is focused on drilling, sampling, and placing instruments to understand seismogenic mechanisms and faulting within the Nankai Trough subduction zone. Recovered sediments are composed of nannofossil-rich hemipelagic mud, silty and sandy turbidites, and sedimentary breccia from slope failures (see the "Expedition 315 summary" chapter [Ashi et al., 2009] and the "Expedition 316 summary" chapter [Screaton et al., 2009]).

In this study we used core samples from Sites C0001, C0004, and C0008 to investigate the megasplay fault and Sites C0006 and C0007, which are located in the frontal thrust region (Fig. F1). Recovered sediments from Site C0001 include slope-apron facies and upper accretionary prism sediments (see the "Expedition 315 Site C0001" chapter [Expedition 315 Scientists, 2009]). Cores from Sites C0004 and C0008 include slope sediments and underthrust slope facies, whereas those from Sites C0006 and C0007 included trench sediments and Shikoku Basin facies sediments (see the "Expedition 316 summary" chapter [Screaton et al., 2009]). Flow-through permeability tests were used to measure the vertical permeability of the samples. To quantify the alignment of grains on faces cut parallel to (vertical) and perpendicular to (horizontal) the core axis, samples for environmental scanning electron microscopy (ESEM) imaging of sediment fabric were located close to 
the whole-round cores and prepared following the general procedures described in Yue et al. (submitted).

\section{Methods}

Permeability tests were conducted using the Trautwein Soil Testing Equipment Company's DigiFlow K (Fig. F2), which consists of a cell (to contain the sample and provide isostatic effective stress) and three pumps (sample top pump, sample bottom pump, and cell pump). Bladder accumulators allow deionized water to be the fluid in the pumps while an idealized solution of seawater $\left(25 \mathrm{~g} \mathrm{NaCl}\right.$ and $8 \mathrm{~g} \mathrm{MgSO}_{4}$ per liter of water) permeates the sample. American Society for Testing and Materials (ASTM) designation D 5084-90 (ASTM International, 1990) was used as a guideline for general procedures.

Core samples from Expeditions 315 and 316 were stored in plastic core liners and sealed in polyester film bags during the cruise after sampling to prevent moisture loss. The sealed samples were stored in the refrigerator at $4^{\circ} \mathrm{C}$ until immediately prior to sample preparation. To provide freshly exposed surfaces, cores were trimmed on both ends using an X-acto knife or a utility knife, depending on core properties. After the ends were trimmed, the diameter and the height of the sample were measured. Samples had a minimum diameter of $2.5 \mathrm{~cm}$, and heights varied from $\sim 3$ to $12 \mathrm{~cm}$. The sample was then placed in a flexible-wall membrane and fitted with saturated porous disks on both ends. Next, the sample was placed in the cell, which was filled with deionized water so that the membrane-encased sample was completely surrounded by fluid. A small confining pressure of $\sim 0.03 \mathrm{MPa}$ (5 psi) was applied, and flow lines were flushed to remove any trapped air bubbles. After the flow lines were flushed, the sample was backpressured to $\sim 0.28 \mathrm{MPa}$ (40 psi) in order to fully saturate it. Backpressure was achieved by concurrently ramping the cell pressure and the sample pressure to maintain a steady effective stress of $0.03 \mathrm{MPa}$. Once the sample reached saturation, the cell fluid pressure was increased while the sample backpressure was maintained, thus increasing the effective stress on the sample. Once the target effective stress was achieved, cell pressure and backpressure were maintained. The sample was allowed to equilibrate for at least $4 \mathrm{~h}$ and generally overnight $(12 \mathrm{~h})$. Throughout testing, inflows and outflows to the cell fluid were monitored to assess changes in sample volume. Because fluid pressure in the closed hydraulic system was affected by temperature changes, testing was conducted within a closed cabinet with a fan to keep the internal temperature uniform. The temperature was maintained at $\sim 30^{\circ} \mathrm{C}\left( \pm 1^{\circ} \mathrm{C}\right)$ during flow tests and consolidation steps. Up to three flow tests were performed at each effective stress level. Flow tests were run by specifying pressures of the top and bottom pump and allowing flow rates into and out of the sample to equilibrate with time.

We used the measured flow rate, cross-sectional area of the sample, and head difference between the top and bottom pumps to calculate hydraulic conductivity using Darcy's law:

$$
Q=-K A(\Delta h / \Delta l),
$$

where

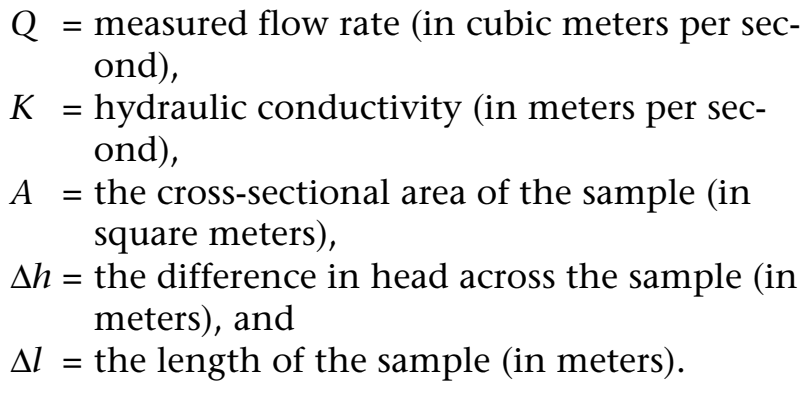

Hydraulic head difference is related to pressure difference $(\Delta P)$ by

$$
\Delta h=\Delta z+\Delta P / \rho g
$$

where

$$
\begin{aligned}
\rho= & \text { fluid density }\left(1020 \mathrm{~kg} / \mathrm{m}^{3}\right) \\
\mathrm{g}= & \text { the gravitational constant }\left(9.81 \mathrm{~m} / \mathrm{s}^{2}\right), \text { and } \\
\Delta Z= & \text { the elevation difference between sample input } \\
& \text { and output locations. }
\end{aligned}
$$

Because the sample was vertical, $\Delta z$ is equal to $\Delta l$, but with the sign depending on the flow direction. The density value was estimated for a temperature of $30^{\circ} \mathrm{C}$ and a salinity of $35 \mathrm{~kg} / \mathrm{m}^{3}$, using the equation developed by Fofonoff (1985). Assuming a reasonable water compressibility, volume change, and thus density change due to the applied pressure, is minor $(<0.1 \%)$.

Hydraulic conductivity values were then converted to permeability (in square meters) using the following equation:

$$
k=(K \mu) /(\rho g),
$$

where $\mu$ is viscosity $(0.0008 \mathrm{~Pa} \cdot \mathrm{s})$.

The viscosity value was obtained from the Handbook of Chemistry and Physics (Lide, 2000) for water at a temperature of $30^{\circ} \mathrm{C}$ and salinity of $35 \mathrm{~kg} / \mathrm{m}^{3}$.

A $2 \mathrm{~h}$ interval of stable flow rates was used for the permeability calculations, and the standard deviation of the permeability during that $2 \mathrm{~h}$ interval was 
calculated to assess uncertainty. Once permeability values were obtained, the cell pressure was increased and the sample was allowed to equilibrate overnight at the new effective stress. For every sample, up to three effective stress steps were performed.

The corresponding porosity for each effective stress was calculated using the change in volume of fluid $(\mathrm{mL})$ contained in the cell during each consolidation step. Total sample volume $\left(V_{\mathrm{T}(0)}\right)$ was calculated using $\pi r^{2} h$, where $r$ is the radius of the core sample and $h$ is the height of the sample. Initial porosities $\left(n_{0}\right)$ for volume calculations were obtained from the "Expedition 316 Site C0004," "Expedition 316 Site C0006," "Expedition 316 Site C0007," and "Expedition 316 Site C0008" chapters (Expedition 316 Scientists, 2009a, 2009b, 2009c, 2009d) and the "Expedition 315 Site C0001" chapter (Expedition 315 Scientists, 2009). We assumed that the porosity of the sample at the end of backpressure is similar to the initial porosity because of the small change in effective stress $(0.03 \mathrm{MPa})$.

Using the initial porosity, the volume of voids before testing $\left(V_{\mathrm{v}(0)}\right)$ was calculated:

$$
V_{\mathrm{v}(0)}=n_{0} V_{\mathrm{T}(0)} .
$$

Volume of solids $\left(V_{\mathrm{s}}\right)$ was calculated using

$$
V_{\mathrm{s}}=V_{\mathrm{T}(0)}-V_{\mathrm{v}(0)} \text {. }
$$

Using the difference of cell volumes between two consecutive steps (e.g., cell volume at backpressure and cell volume at first consolidation), the change in volume of water in the cell $\left(\Delta V_{\mathrm{T}(1)}\right)$ was calculated. The new total volume of the sample $\left(V_{\mathrm{T}(1)}\right)$ after pore spaces were reduced during the consolidation process was determined by subtracting the change in cell volume at the end of the consolidation step from the total sample volume:

$$
V_{\mathrm{T}(1)}=V_{\mathrm{T}(0)}-\Delta V_{\mathrm{T}(1)} .
$$

Using the calculated new total volume of the sample, the new porosity at the end of the consolidation is calculated. The new porosity $\left(n_{1}\right)$ at the end of the consolidation is

$$
n_{1}=\left(1-V_{\mathrm{s}}\right) / V_{\mathrm{T}(1)} .
$$

The greatest error in porosity estimates is expected to be due to loss of water from the cell during testing because of minor leaks in the equipment. Loss of water was evaluated by examining inflow to the cell during flow tests, when effective stress was stable and sample volume should not be changing. These loss rates were then used to estimate the porosity un- derestimate. The actual error may be less because samples may continue to slowly consolidate during the flow tests. The average volume lost during a sample run was $5.0 \mathrm{~mL}$, and the maximum estimated loss was $17 \mathrm{~mL}$. The estimated errors in porosities are displayed as error bars on Figure F3 and are highly dependent on initial volume of voids (and thus sample volume). For most whole-round samples, the potential porosity error was $\sim 0.025$. For $2.54 \mathrm{~cm}$ (1 inch) diameter subsamples, however, the volume error was the same order of magnitude as the initial void volume, preventing reliable estimation of porosity change.

The approach used for fabric quantification follows the statistics of Folk and Ward (1957) for standard deviation, as described in Yue et al. (submitted). The standard deviation of orientation (d) equals

$$
d=\left[\left(\phi_{84}-\phi_{16}\right) / 4\right]+\left[\left(\phi_{95}-\phi_{5}\right) / 6.6\right]
$$

where $\phi_{84}, \phi_{16}, \phi_{95}$, and $\phi_{5}$ represent the angle of orientation of a particle's apparent long axis at the 84th, 16th, 95th, and 5th percentiles, respectively, on the cumulative frequency curve. This graphical technique avoids the laborious calculations required by moment statistics (Chiou et al., 1991). Numerically, the largest value of $d$ is $72.3^{\circ}$ (i.e., a case in which $\phi_{5}$ and $\phi_{16}=0^{\circ}$ and $\phi_{84}$ and $\phi_{95}=180^{\circ}$ ). We normalized each standard deviation to this maximum $d$ value by calculating the "index of microfabric orientation" (i) as

$$
i=1-(d / 72.3) \text {. }
$$

The closer the value of $i$ is to 1 , the more the particles are aligned in a preferred direction.

For a random arrangement of particles, the cumulative curve displays a gentle slope near the median (50th percentile), the standard deviation of orientation is $>35^{\circ}$, and the index of microfabric orientation is $<0.51$. For highly oriented clay particles, the slope of the cumulative curve is generally $>1.00$ near the median, the standard deviation of grain orientation is $<25^{\circ}$, and the index of microfabric orientation is $>0.65$. We also report fabric results using rose diagrams to illustrate the number of grains (apparent long axes) within each $10^{\circ}$ orientation bin for surfaces cut parallel to the core axis (vertical section) and perpendicular to the core axis (horizontal section).

\section{Results}

Table T1 summarizes the effective stress and estimated porosity and permeability at each consolida- 
tion step. In general, permeabilities decreased with increasing effective stress. Permeability is plotted as a function of porosity in Figure F3. Tests were attempted on five additional whole-round samples (315-C0001E-10H-3, 0.0-20.0 cm; 315-C0001F-11H-7, 101.0-116.0 cm; 315-C0001H-23R-3, 0.0-20.0 cm; $316-\mathrm{C} 0006 \mathrm{~F}-10 \mathrm{R}-1,16.5-27.5 \mathrm{~cm}$; and 316-C0004C$6 \mathrm{H}-7,0.0-15.0 \mathrm{~cm}$ ) but were unsuccessful because of poor sample condition or irregular geometry. The measured permeabilities vary from $\sim 2.51 \times 10^{-19} \mathrm{~m}^{2}$ to $\sim 5.26 \times 10^{-16} \mathrm{~m}^{2}$. In general, the lower permeabilities tend to represent clay-rich sediments, whereas the higher permeabilities represent sand-rich, interbedded ash sediments or fractured samples.

Table T2 summarizes the details of ESEM fabric analysis results. Some of the samples used for permeability did not have adjacent samples for ESEM, and some ESEM samples displayed do not have permeability results, generally because of sample condition. Rose diagrams in Figure F4 show the orientations of the fabrics. The cumulative curves are shown in Figure $\mathbf{F 5}$. The average index of microfabric orientation is $\sim 0.30-0.34$. Most vertical sections show greater indexes of orientation and steeper cumulative slopes than the horizontal sections for samples from the slope layer at Sites C0001, C0004, and C0008 (Table T2; Fig. F5). Some samples from the accreted wedge at Site C0001, slope sediments in the footwall of the megasplay fault at Site C0004, and frontal thrust Sites C0006 and C0007 exhibit higher orientation indexes (0.37-0.54) and concomitant steeper cumulative slopes than other specimens (Table T2; Fig. F5), indicating a higher general alignment of grains.

\section{Acknowledgments}

This research used samples and data provided by the Integrated Ocean Drilling Program. Funding for this research was provided by the Consortium for Ocean Leadership U.S. Scientist Support Program postcruise grants to E. Screaton (T316A28) and M. Underwood (T315B58 and T315C58) and National Science Foundation grants to E. Screaton (OCE-0751497) and M. Underwood (OCE-0751819). Review by Glenn Spinelli improved this manuscript.

\section{References}

Ashi, J., Lallemant, S., Masago, H., and the Expedition 315 Scientists, 2009. Expedition 315 summary. In Kinoshita, M., Tobin, H., Ashi, J., Kimura, G., Lallemant, S., Screaton, E.J., Curewitz, D., Masago, H., Moe, K.T., and the Expedition 314/315/316 Scientists, Proc. IODP, 314/315/ 316: Washington, DC (Integrated Ocean Drilling Program Management International, Inc.). doi:10.2204/ iodp.proc.314315316.121.2009
ASTM International, 1990. Standard test method for measurement of hydraulic conductivity of saturated porous materials using a flexible wall permeameter (Standard D5084-70). In Annual Book of ASTM Standards: Philadelphia (Am. Soc. Testing Mater.), 63-70.

Chiou, W.-A., Bennett, R.H., and Bryant, W.R., 1991. Quantification of clay fabric: a simple technique. In Bennett, R.H., Bryant, W.R., and Hulbert, M.H. (Eds.), Microstructure of Fine-Grained Sediments: From Mud to Shale: New York (Springer-Verlag), 379-387.

Expedition 315 Scientists, 2009. Expedition 315 Site C0001. In Kinoshita, M., Tobin, H., Ashi, J., Kimura, G., Lallemant, S., Screaton, E.J., Curewitz, D., Masago, H., Moe, K.T., and the Expedition 314/315/316 Scientists, Proc. IODP, 314/315/316: Washington, DC (Integrated Ocean Drilling Program Management International, Inc.). doi:10.2204/iodp.proc.314315316.123.2009

Expedition 316 Scientists, 2009a. Expedition 316 Site C0004. In Kinoshita, M., Tobin, H., Ashi, J., Kimura, G., Lallemant, S., Screaton, E.J., Curewitz, D., Masago, H., Moe, K.T., and the Expedition 314/315/316 Scientists, Proc. IODP, 314/315/316: Washington, DC (Integrated Ocean Drilling Program Management International, Inc.). doi:10.2204/iodp.proc.314315316.133.2009

Expedition 316 Scientists, 2009b. Expedition 316 Site C0006. In Kinoshita, M., Tobin, H., Ashi, J., Kimura, G., Lallemant, S., Screaton, E.J., Curewitz, D., Masago, H., Moe, K.T., and the Expedition 314/315/316 Scientists, Proc. IODP, 314/315/316: Washington, DC (Integrated Ocean Drilling Program Management International, Inc.). doi:10.2204/iodp.proc.314315316.134.2009

Expedition 316 Scientists, 2009c. Expedition 316 Site C0007. In Kinoshita, M., Tobin, H., Ashi, J., Kimura, G., Lallemant, S., Screaton, E.J., Curewitz, D., Masago, H., Moe, K.T., and the Expedition 314/315/316 Scientists, Proc. IODP, 314/315/316: Washington, DC (Integrated Ocean Drilling Program Management International, Inc.). doi:10.2204/iodp.proc.314315316.135.2009

Expedition 316 Scientists, 2009d. Expedition 316 Site C0008. In Kinoshita, M., Tobin, H., Ashi, J., Kimura, G., Lallemant, S., Screaton, E.J., Curewitz, D., Masago, H., Moe, K.T., and the Expedition 314/315/316 Scientists, Proc. IODP, 314/315/316: Washington, DC (Integrated Ocean Drilling Program Management International, Inc.). doi:10.2204/iodp.proc.314315316.136.2009

Fofonoff, N.P., 1985. Physical properties of seawater: a new salinity scale and equation of state for seawater. J. Geophys. Res., [Oceans], 90(C2):3332-3342. doi:10.1029/ JC090iC02p03332

Folk, R.L., and Ward, W.C., 1957. Brazos River bar [Texas]: a study in the significance of grain size parameters. $J$. Sediment. Res., 27(1):3-26. http://jsedres.sepmonline.org/cgi/content/abstract/27/1/3

Gamage, K., and Screaton, E., 2006. Characterization of excess pore pressures at the toe of the Nankai accretionary complex, Ocean Drilling Program Sites 1173, 1174, and 808: results of one-dimensional modeling. J. Geophys. Res., [Solid Earth], 111(B4):B04103-B05115. doi:10.1029/2004JB003572 
Lide, D.R. (Ed.), 2000. Handbook of Chemistry and Physics: Boca Raton, FL (Chemical Rubber Publishing Company).

Moore, G.F., Park, J.-O., Bangs, N.L., Gulick, S.P., Tobin, H.J., Nakamura, Y., Sato, S., Tsuji, T., Yoro, T., Tanaka, H., Uraki, S., Kido, Y., Sanada, Y., Kuramoto, S., and Taira, A., 2009. Structural and seismic stratigraphic framework of the NanTroSEIZE Stage 1 transect. In Kinoshita, M., Tobin, H., Ashi, J., Kimura, G., Lallemant, S., Screaton, E.J., Curewitz, D., Masago, H., Moe, K.T., and the Expedition 314/315/316 Scientists, Proc. IODP, 314/315/316: Washington, DC (Integrated Ocean Drilling Program Management International, Inc.). doi:10.2204/iodp.proc.314315316.102.2009

Screaton, E.J., Kimura, G., Curewitz, D., and the Expedition 316 Scientists, 2009. Expedition 316 summary. In Kinoshita, M., Tobin, H., Ashi, J., Kimura, G., Lallemant, S., Screaton, E.J., Curewitz, D., Masago, H., Moe,
K.T., and the Expedition 314/315/316 Scientists, Proc. IODP, 314/315/316: Washington, DC (Integrated Ocean Drilling Program Management International, Inc.). doi:10.2204/iodp.proc.314315316.131.2009

Yue, L., Likos, W.J., Guo, J., and Underwood, M.B., submitted. Data report: permeability of mud(stone) samples from Site C0001, IODP Expedition 315, Nankai Trough: Stage 1 of NanTroSEIZE. In Kinoshita, M., Tobin, H., Ashi, J., Kimura, G., Lallemant, S., Screaton, E.J., Curewitz, D., Masago, H., Moe, K.T., and the Expedition 314/ 315/316 Scientists, Proc. IODP, 314/315/316: Washington, DC (Integrated Ocean Drilling Program Management International, Inc.).

Initial receipt: 18 October 2010

Acceptance: 7 September 2011

Publication: 8 November 2011

MS 314315316-211 
Figure F1. Location map for Expeditions 315 and 316 (modified from Moore et al., 2009). Samples for this study were collected from Sites C0001, C0004, C0006, C0007, and C0008. Locations of previous Nankai Trough drilling (Ashizuri and Muroto transects) are also shown. KPR $=$ Kyushu-Palau Ridge, FSC $=$ fossil spreading center, PSP = Philippine Sea plate, IBT = Izu-Bonin Trench.

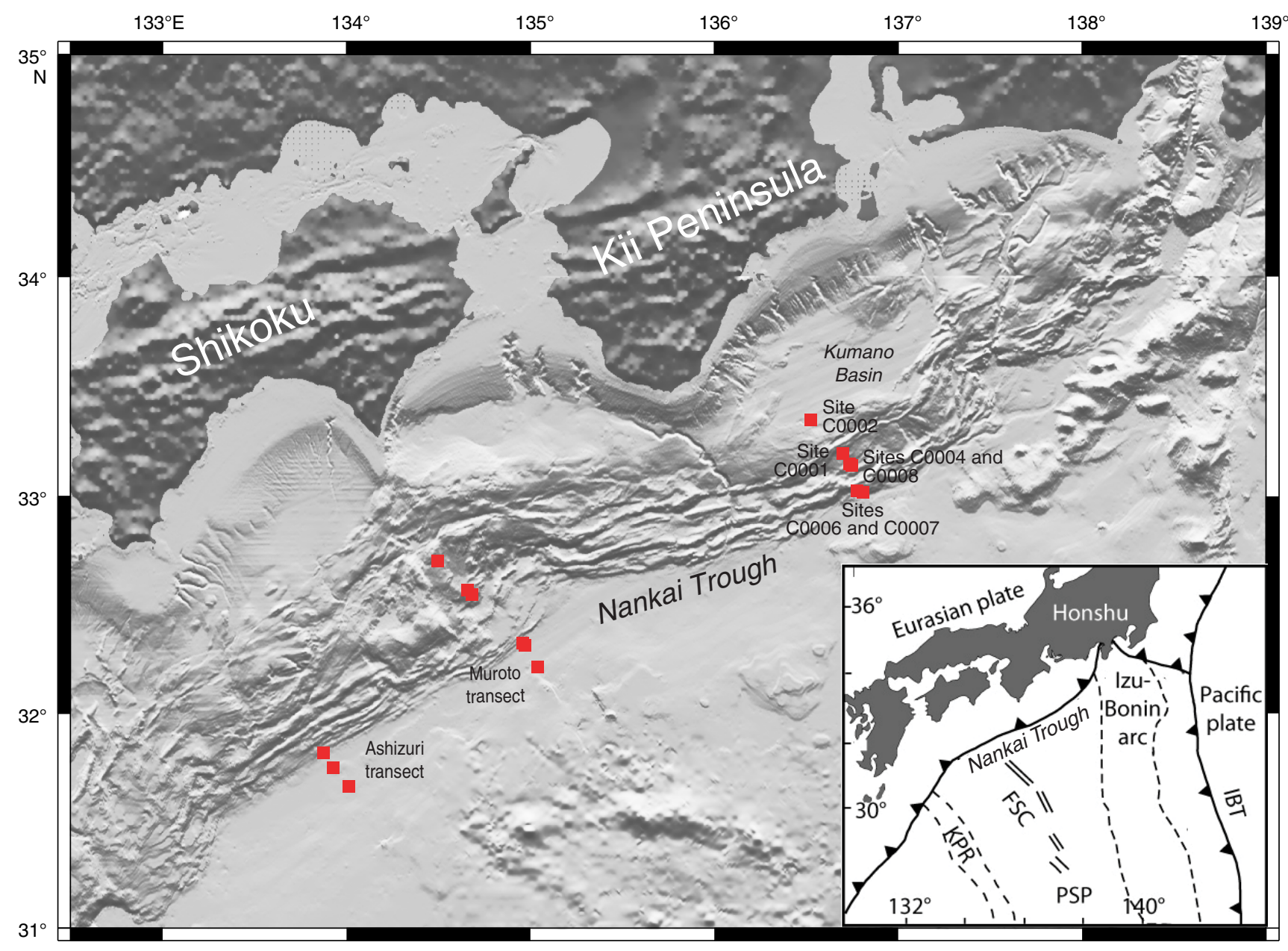


Figure F2. Schematic of permeability test system.

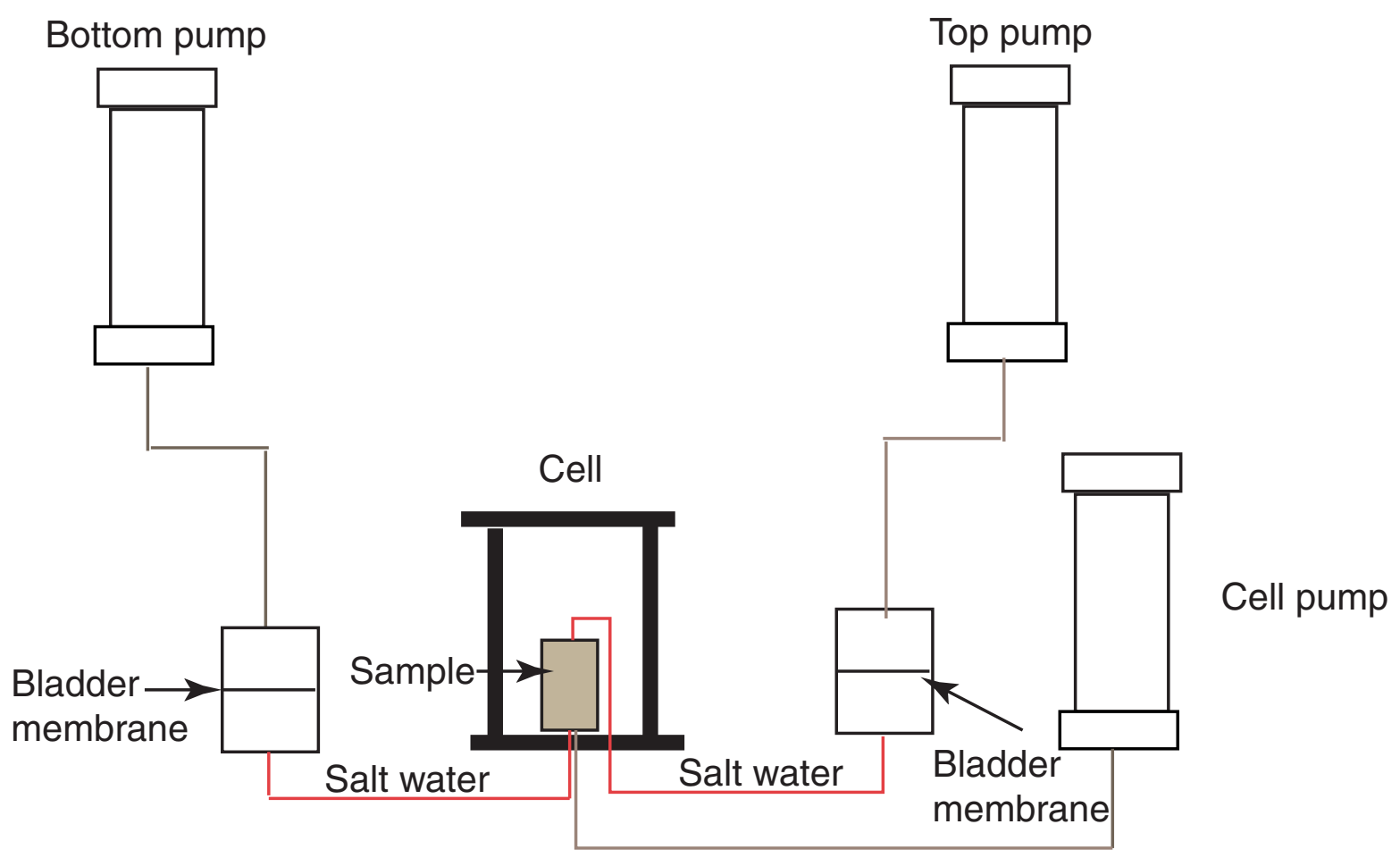


Figure F3. Plot of measured permeability as a function of porosity, Sites C0001, C0004, C0006, C0007, and C0008. Open symbols $=$ samples containing sand or ash, solid symbols $=$ more clayey samples. ${ }^{*}=2.54 \mathrm{~cm}(1$ inch) diameter samples. Porosity change due to consolidation could not be estimated for these samples; permeability from the first consolidation step is shown plotted against initial (shipboard) porosity. For comparison, the inferred porosity permeability relationship $(\log (k)=19.82+5.39 n)$ from the Muroto transect (Gamage and Screaton, 2006) is shown.

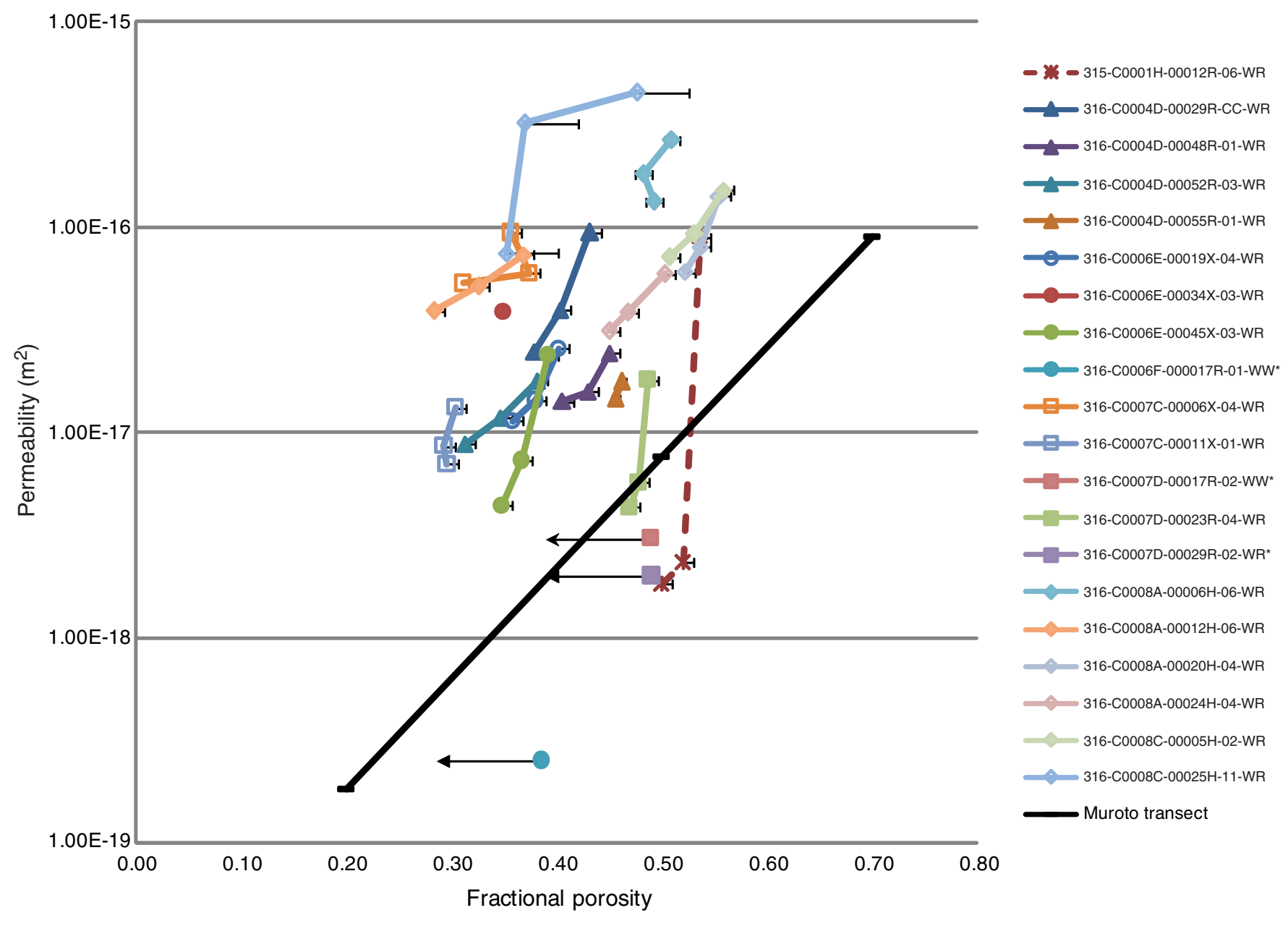


Figure F4. Rose diagrams showing fabric orientations of samples from Sites C0001, C0004, C0006, C0007, and C0008. The specific orientation of each particle (apparent long axis) was assigned an angle between $0^{\circ}$ and $180^{\circ}$. A. Site C0001. (Continued on next four pages.)
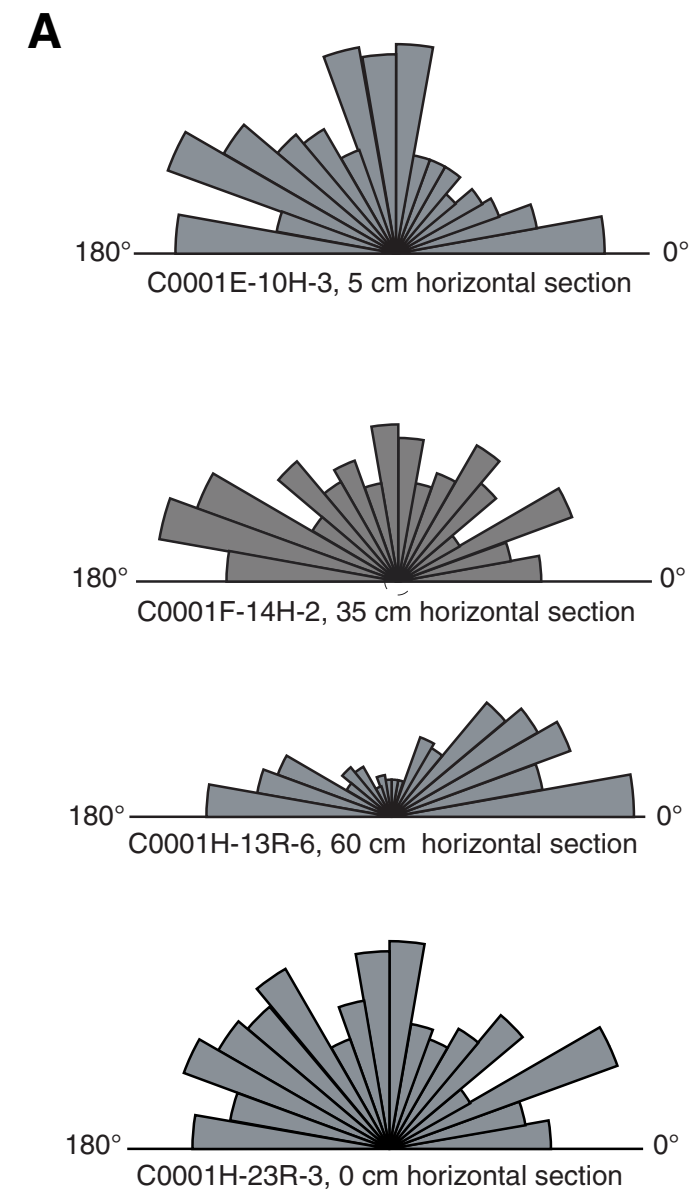
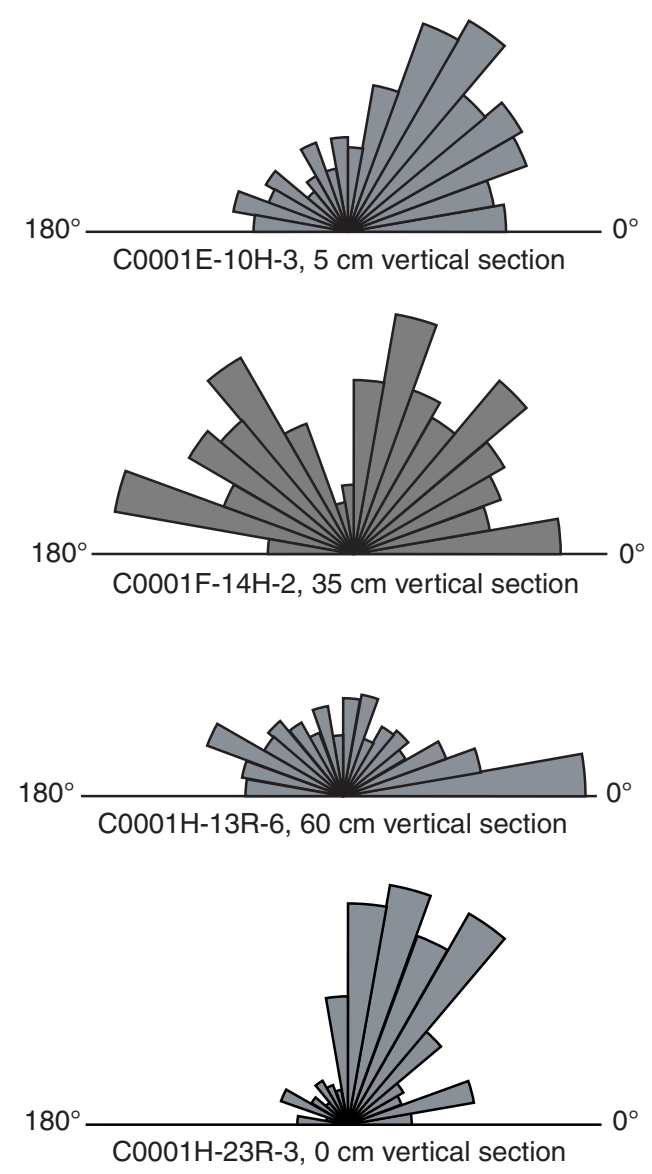
Figure F4 (continued). B. Site C0004. (Continued on next page.)

B
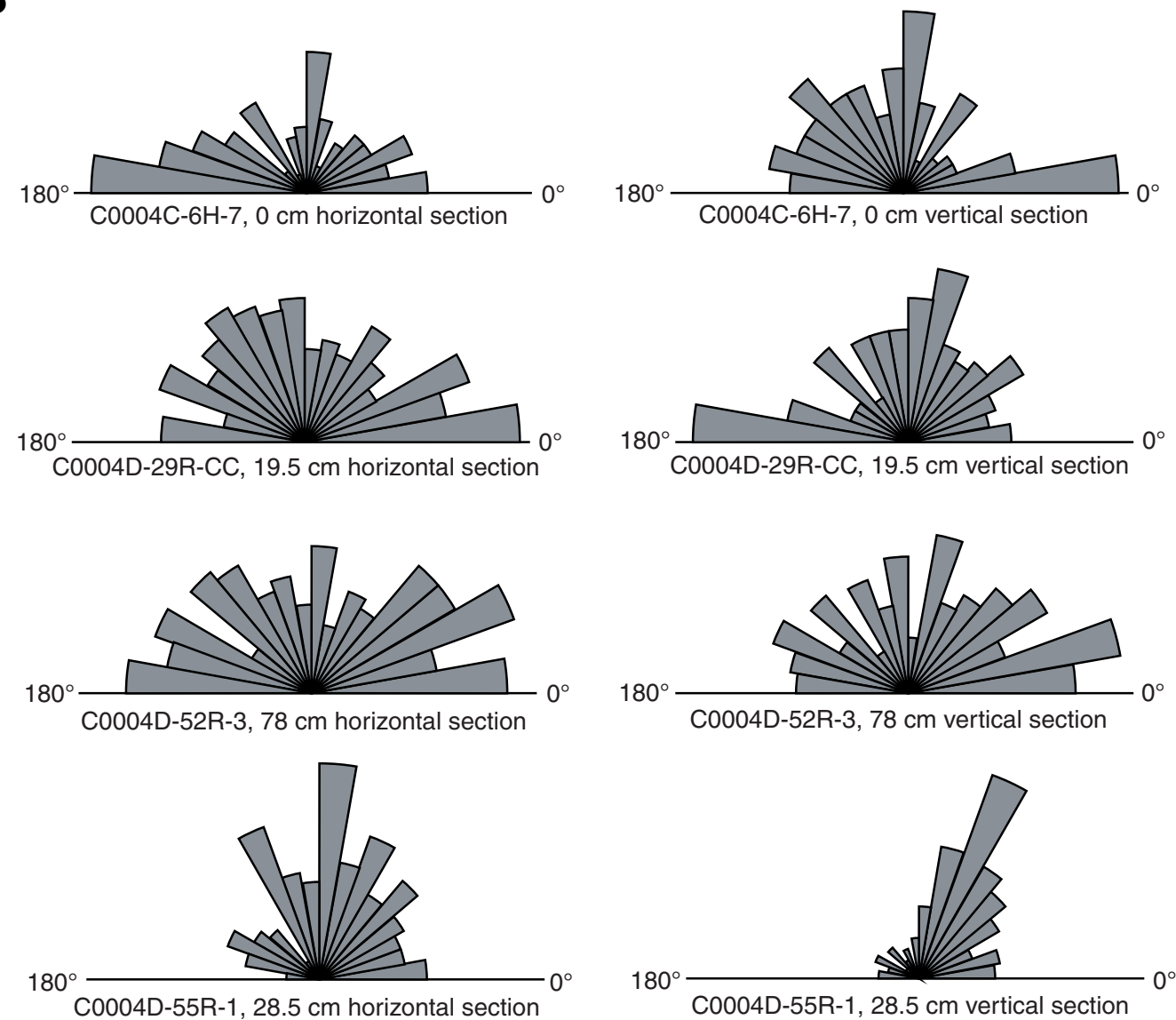
Figure F4 (continued). C. Site C0006. (Continued on next page.)

C
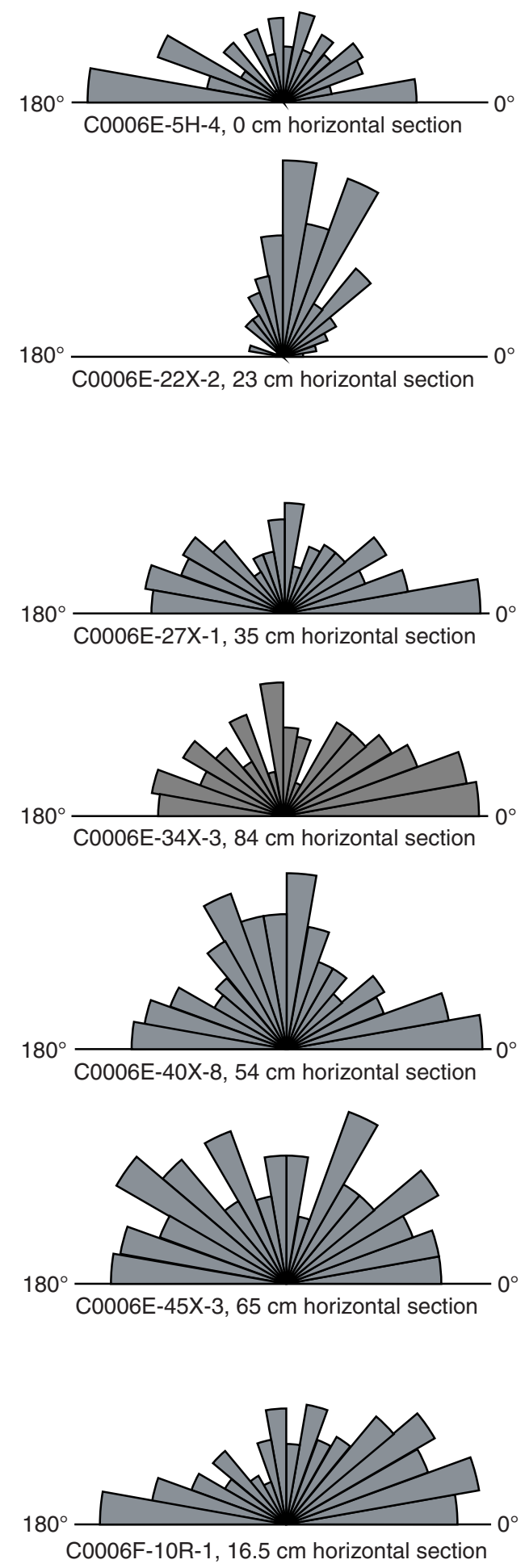
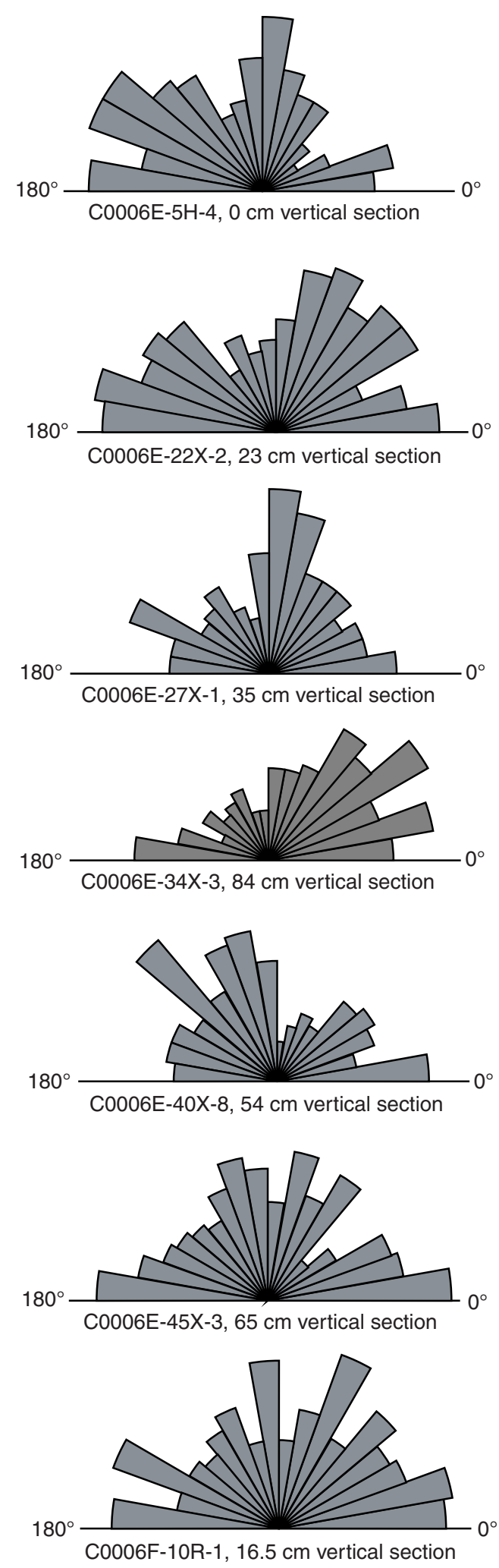
Figure F4 (continued). D. Site C0007. (Continued on next page.)

D

$180^{\circ}$
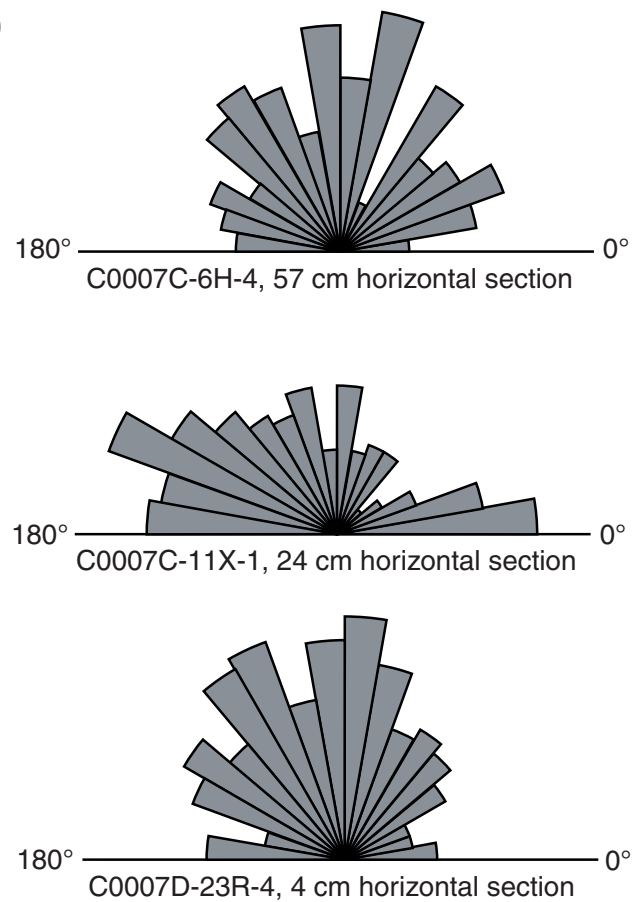
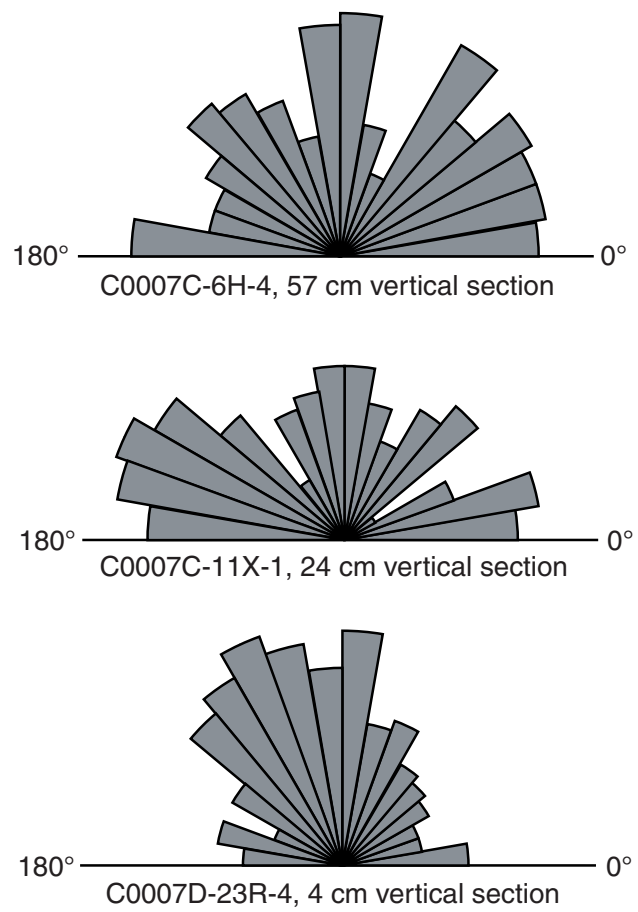
Figure F4 (continued). E. Site C0008.

E
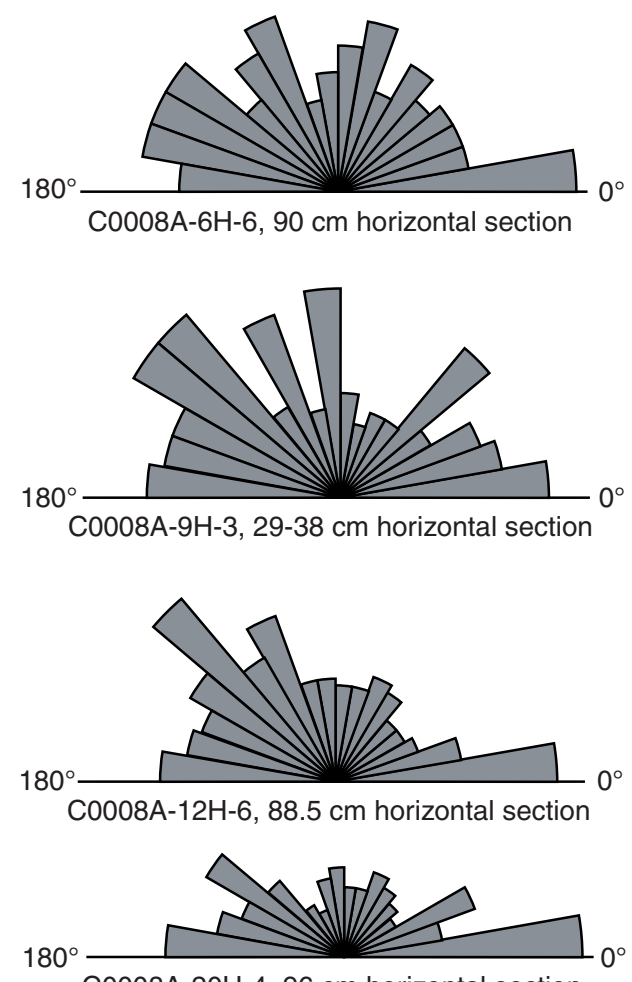

C0008A-20H-4, $96 \mathrm{~cm}$ horizontal section
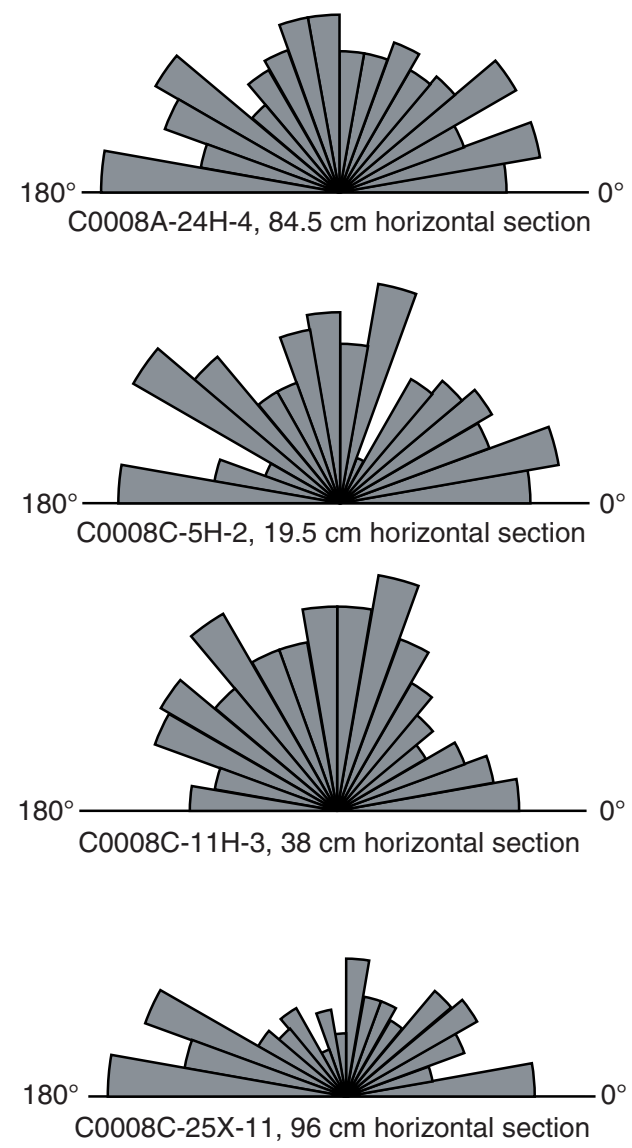
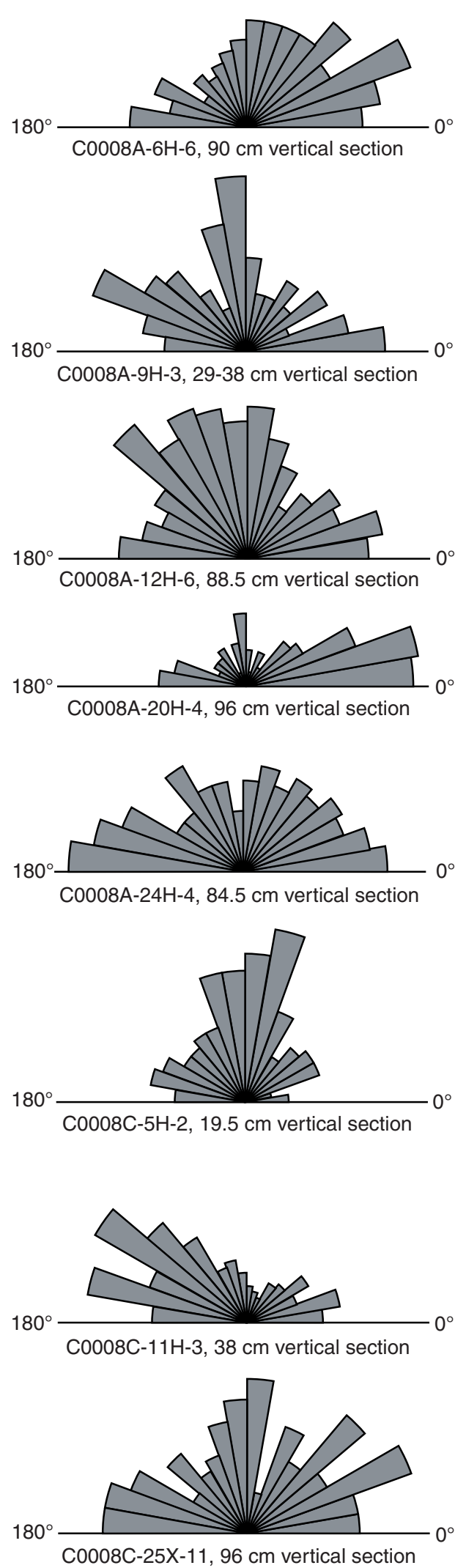
Figure F5. Cumulative orientation frequency curve of samples from Sites C0001, C0004, C0006, C0007, and C0008. The number of values in each bin $\left(10^{\circ}\right)$ was summed and normalized to $100 \%$. A. Site C0001. (Continued on next four pages.)

A

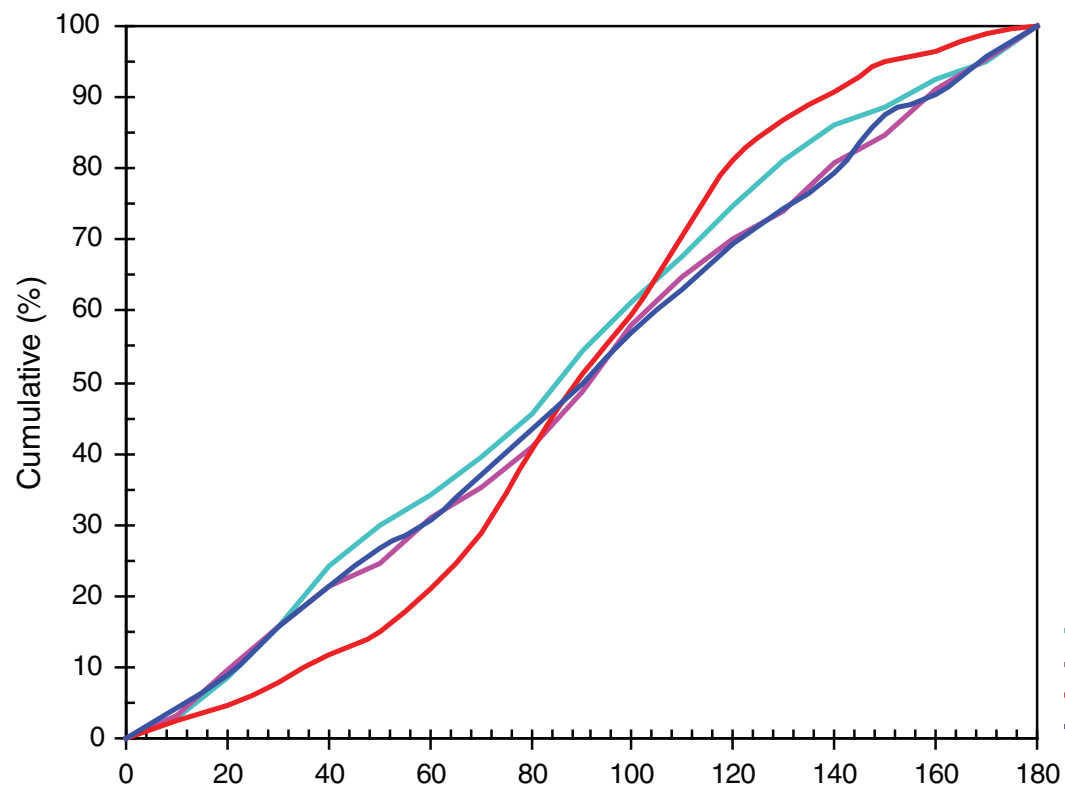

$\mathrm{C} 0001 \mathrm{E}-10 \mathrm{H}-3,0 \mathrm{~cm}$

$\mathrm{C} 0001 \mathrm{~F}-14 \mathrm{H}-2,35 \mathrm{~cm}$ C0001H-13R-6, $60 \mathrm{~cm}$

Orientation of particles in section cut perpendicular to core axis $\left(^{\circ}\right)$

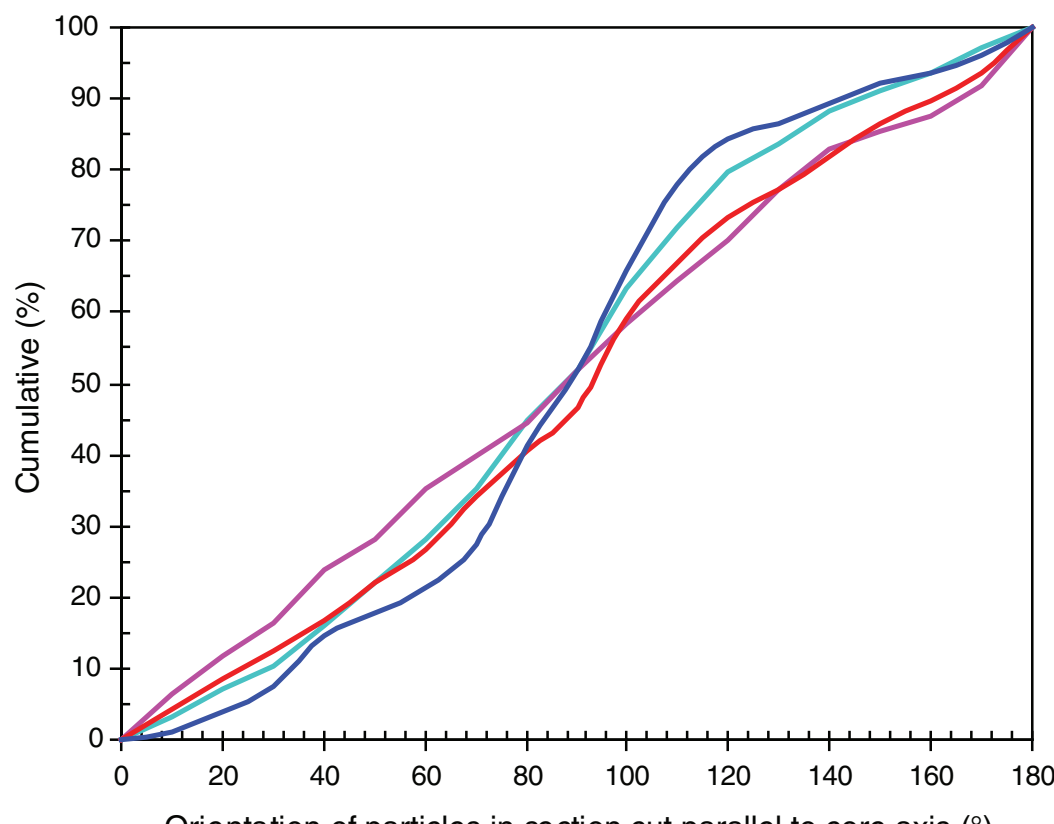

C0001E-10H-3, $0 \mathrm{~cm}$

C0001F-14H-2, $35 \mathrm{~cm}$

$\mathrm{C} 0001 \mathrm{H}-13 \mathrm{R}-6,60 \mathrm{~cm}$

Orientation of particles in section cut parallel to core axis $\left(^{\circ}\right)$ 
Figure F5 (continued). B. Site C0004. (Continued on next page.)

B
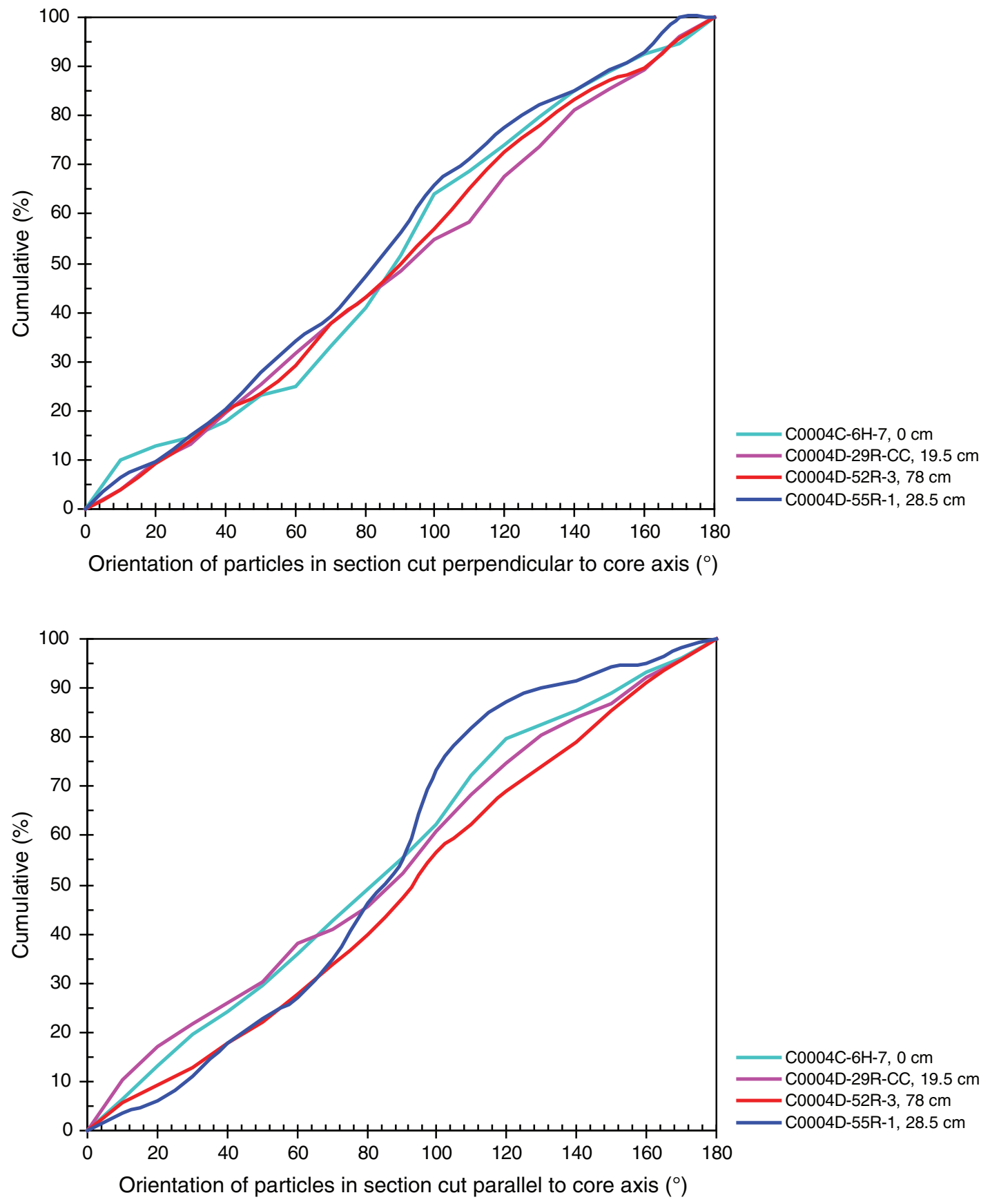
Figure F5 (continued). C. Site C0006. (Continued on next page.)

C

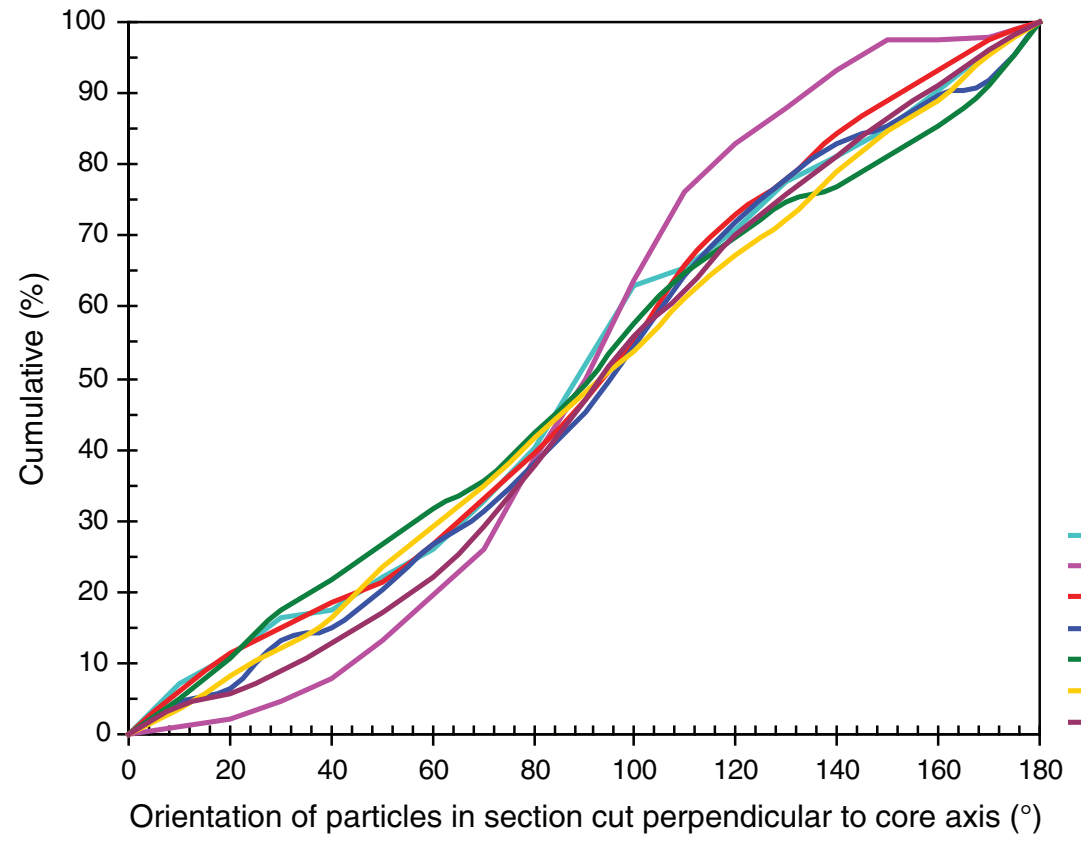

C0006E-5H-4, $0 \mathrm{~cm}$ C0006E-22X-2, $23 \mathrm{~cm}$ C0006E-27X-1, $35 \mathrm{~cm}$ C0006E-34X-3, $84 \mathrm{~cm}$ C0006E-40X-8, $54 \mathrm{~cm}$ 0006E-45X-3, $65 \mathrm{~cm}$ C0006F-10R-1, $16.5 \mathrm{~cm}$

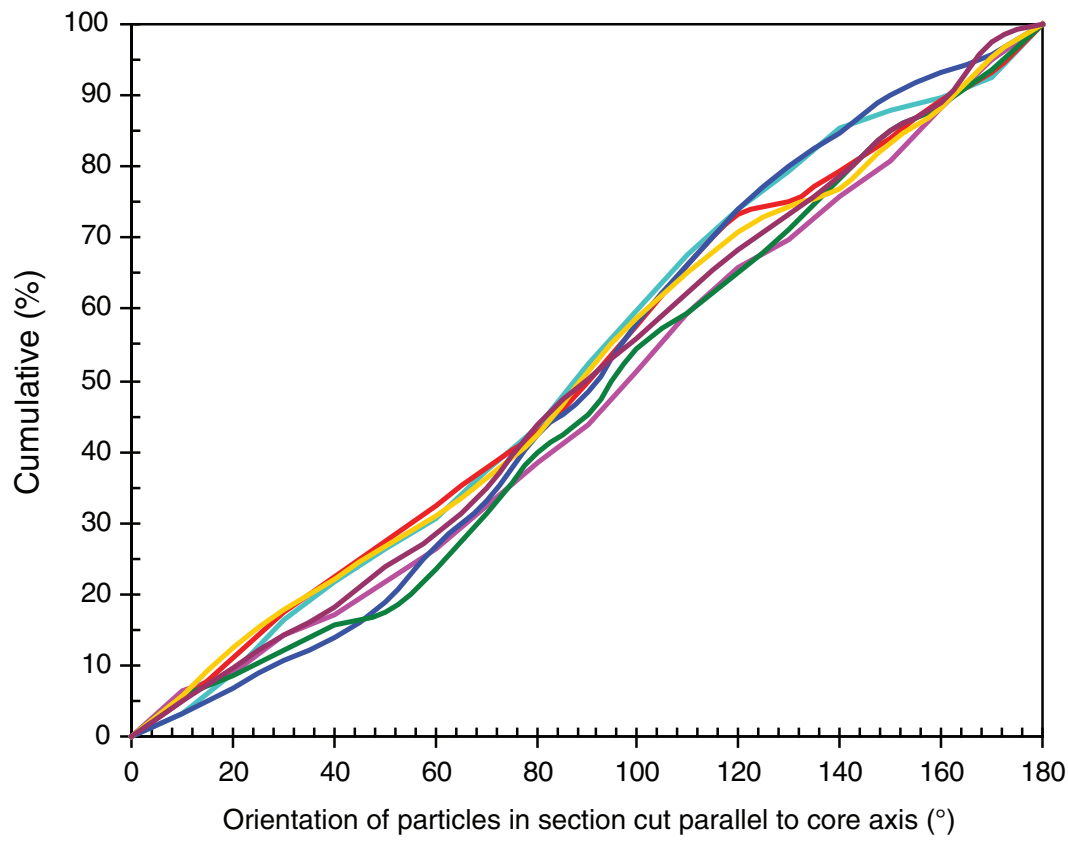

C0006E-5H-4, $0 \mathrm{~cm}$

C0006E-22X-2, $23 \mathrm{~cm}$

C0006E-27X-1, $35 \mathrm{~cm}$

C0006E-34X-3, $84 \mathrm{~cm}$

C0006E-40X-8, $54 \mathrm{~cm}$ C0006E-45X-3, $65 \mathrm{~cm}$ C0006F-10R-1, $16.5 \mathrm{~cm}$ 
Figure F5 (continued). D. Site C0007. (Continued on next page.)

D
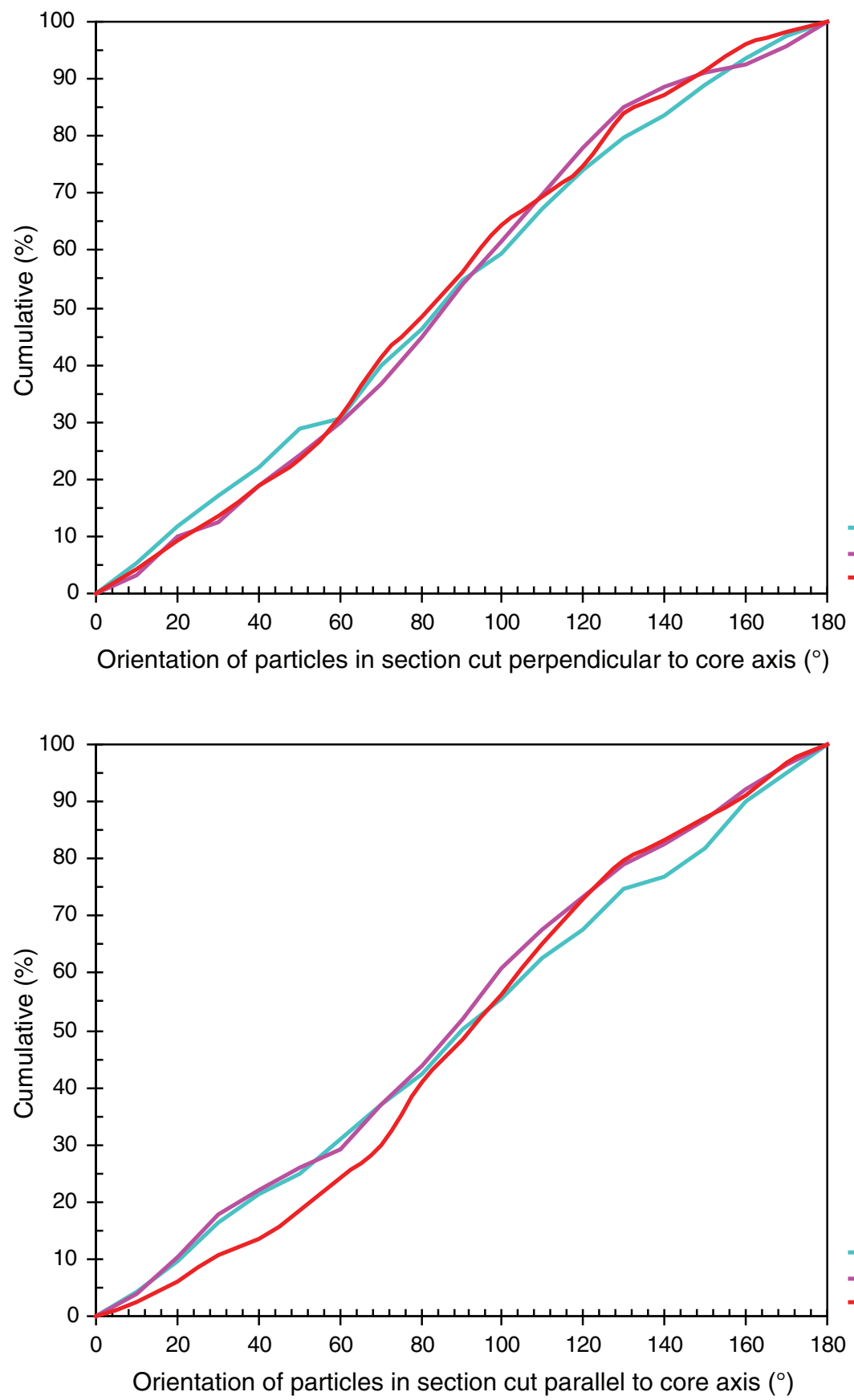

C0007C-6X-4, $57 \mathrm{~cm}$ 
Figure F5 (continued). E. Site C0008.

E

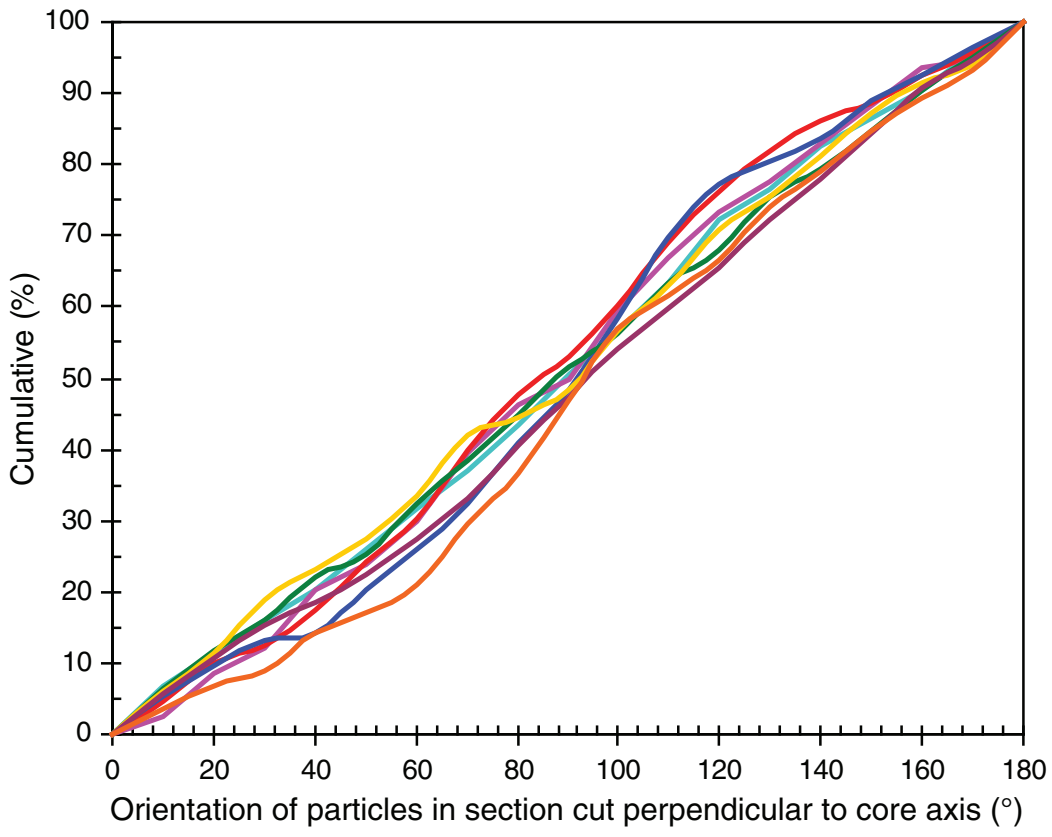

C0008A-6H-6, $90 \mathrm{~cm}$ C0008A-9H-3, 29-38 cm C0008A-12H-6, $88.5 \mathrm{~cm}$ $\mathrm{C} 0008 \mathrm{~A}-2 \mathrm{OH}-4,96 \mathrm{~cm}$ C0008A-24H-4, $84.5 \mathrm{~cm}$ C0008C-5H-2, $19.5 \mathrm{~cm}$ $\mathrm{C} 0008 \mathrm{C}-11 \mathrm{H}-3,38 \mathrm{~cm}$ C0008C-25X-11, $96 \mathrm{~cm}$

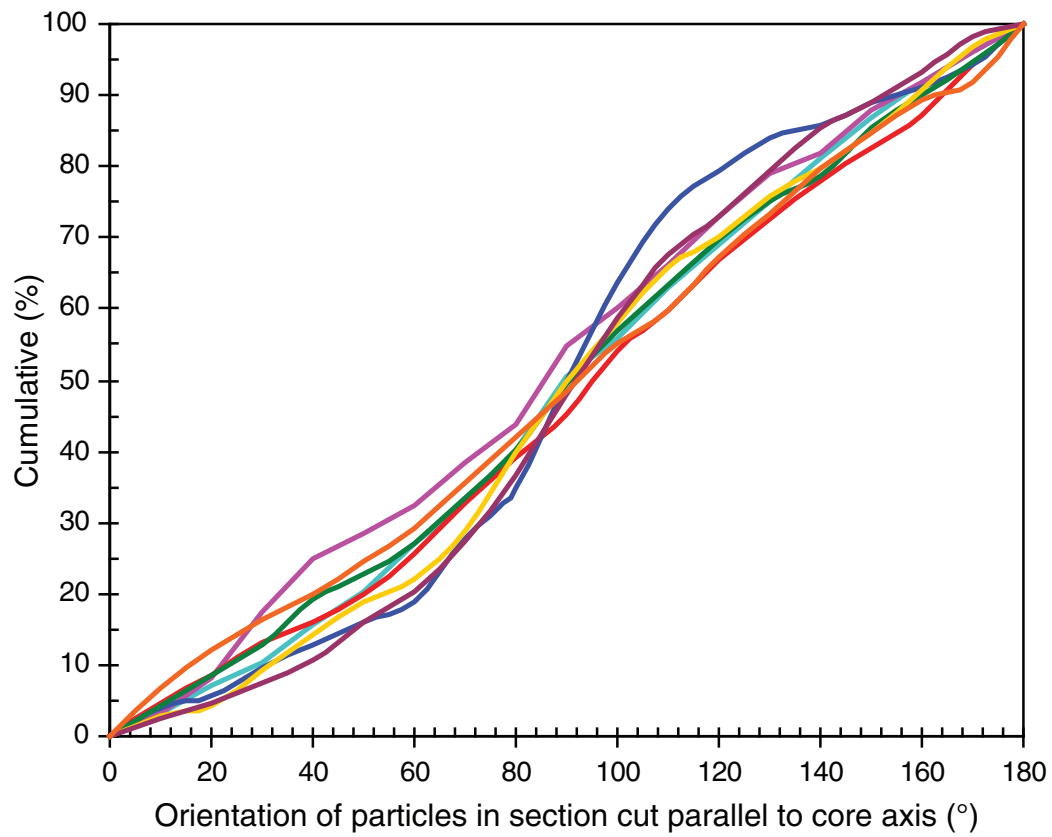

C0008A-6H-6, $90 \mathrm{~cm}$ C0008A-9H-3, 29-38 cm C0008A-12H-6, $88.5 \mathrm{~cm}$ $\mathrm{C} 0008 \mathrm{~A}-2 \mathrm{OH}-4,96 \mathrm{~cm}$ C0008A-24H-4, $84.5 \mathrm{~cm}$ C0008C-5H-2, $19.5 \mathrm{~cm}$ $\mathrm{C} 0008 \mathrm{C}-11 \mathrm{H}-3,38 \mathrm{~cm}$ C0008C-25X-11, $96 \mathrm{~cm}$ 
Table T1. Results from laboratory permeability tests, Sites C0001, C0004, C0006, C0007, and C0008. (Continued on next four pages.)

\begin{tabular}{|c|c|c|c|c|c|c|c|c|c|c|c|c|c|}
\hline $\begin{array}{l}\text { Core, section, } \\
\text { interval }(\mathrm{cm})\end{array}$ & $\begin{array}{l}\text { Depth } \\
\text { (mbsf) }\end{array}$ & $\begin{array}{l}\text { Core } \\
\text { diameter } \\
(\mathrm{m})\end{array}$ & $\begin{array}{c}\text { Sample } \\
\text { length } \\
\text { (m) }\end{array}$ & Lithology & $\begin{array}{l}\text { Initial } \\
\text { porosity } \\
(\%)\end{array}$ & Run & $\begin{array}{c}\text { Effective } \\
\text { stress } \\
(\mathrm{MPa})\end{array}$ & $\begin{array}{l}\text { Flow rate } \\
(\mathrm{mL} / \mathrm{min})\end{array}$ & $\begin{array}{l}\text { Fractional } \\
\text { porosity }\end{array}$ & $\begin{array}{c}\Delta \text { head } \\
(\mathrm{m})\end{array}$ & $\underset{(\mathrm{m} / \mathrm{s})}{K}$ & $\begin{array}{c}k \\
\left(m^{2}\right)\end{array}$ & $\begin{array}{c}k \\
\text { standard } \\
\text { deviation } \\
\left(\mathrm{m}^{2}\right)\end{array}$ \\
\hline \multicolumn{14}{|l|}{ 315-C0001H- } \\
\hline \multirow[t]{12}{*}{ 13R-6-WR, 60-80 } & \multirow[t]{12}{*}{346.75} & \multirow[t]{12}{*}{0.056} & \multirow[t]{12}{*}{0.136} & \multirow[t]{12}{*}{ Silty clay } & \multirow[t]{12}{*}{54.59} & 1 & 0.14 & $8.59 \mathrm{E}-03$ & \multirow[t]{4}{*}{0.54} & 1.48 & $3.88 \mathrm{E}-10$ & $3.11 \mathrm{E}-17$ & $2.90 \mathrm{E}-19$ \\
\hline & & & & & & 2 & 0.14 & $1.32 \mathrm{E}-02$ & & 2.17 & 4.07E-10 & $3.26 \mathrm{E}-17$ & 1.17E-18 \\
\hline & & & & & & 3 & 0.14 & $9.68 \mathrm{E}-02$ & & 2.56 & $2.54 \mathrm{E}-09$ & $2.03 \mathrm{E}-16$ & $4.03 \mathrm{E}-18$ \\
\hline & & & & & & Averages: & 0.14 & $3.95 \mathrm{E}-02$ & & & $1.11 \mathrm{E}-09$ & $8.89 \mathrm{E}-17$ & \\
\hline & & & & & & 1 & 0.27 & $6.16 \mathrm{E}-04$ & \multirow[t]{4}{*}{0.52} & 1.50 & $2.76 \mathrm{E}-11$ & $2.21 \mathrm{E}-18$ & \\
\hline & & & & & & 2 & 0.27 & 8.43E-04 & & 1.93 & $2.94 \mathrm{E}-11$ & 2.35E-18 & $1.56 \mathrm{E}-20$ \\
\hline & & & & & & 3 & 0.27 & $1.26 \mathrm{E}-03$ & & 2.87 & $2.94 \mathrm{E}-11$ & $2.35 \mathrm{E}-18$ & 2.87E-20 \\
\hline & & & & & & Averages: & 0.27 & $9.06 \mathrm{E}-04$ & & & $2.88 \mathrm{E}-11$ & $2.30 \mathrm{E}-18$ & $1.35 \mathrm{E}-20$ \\
\hline & & & & & & 1 & 0.41 & $4.85 \mathrm{E}-04$ & \multirow[t]{4}{*}{0.50} & 1.25 & $2.61 \mathrm{E}-11$ & $2.09 \mathrm{E}-18$ & \\
\hline & & & & & & 2 & 0.41 & $6.00 \mathrm{E}-04$ & & 2.19 & $1.84 \mathrm{E}-11$ & $1.47 \mathrm{E}-18$ & $1.33 \mathrm{E}-19$ \\
\hline & & & & & & 3 & 0.41 & $9.19 \mathrm{E}-04$ & & 2.62 & $2.36 \mathrm{E}-11$ & $1.88 \mathrm{E}-18$ & $1.14 \mathrm{E}-19$ \\
\hline & & & & & & Averages: & 0.41 & $6.68 \mathrm{E}-04$ & & & $2.27 \mathrm{E}-11$ & $1.81 \mathrm{E}-18$ & 1.97E-19 \\
\hline \multirow{13}{*}{$\begin{array}{l}\text { 316-C0004D- } \\
\text { 29R-CC-WR, 19.5-25.5 }\end{array}$} & \multirow{13}{*}{277.14} & \multirow{13}{*}{0.062} & \multirow{13}{*}{0.041} & \multirow{13}{*}{ Silty claystone } & & & & & & & & & \\
\hline & & & & & \multirow[t]{12}{*}{46.00} & 1 & 0.14 & $2.68 \mathrm{E}-02$ & \multirow[t]{4}{*}{0.43} & 1.09 & $1.36 \mathrm{E}-09$ & $1.09 \mathrm{E}-16$ & $3.26 \mathrm{E}-18$ \\
\hline & & & & & & 2 & 0.14 & $4.08 \mathrm{E}-02$ & & 2.21 & $1.02 \mathrm{E}-09$ & $8.16 \mathrm{E}-17$ & $2.16 \mathrm{E}-18$ \\
\hline & & & & & & 3 & 0.14 & $4.90 \mathrm{E}-02$ & & 2.43 & $1.11 \mathrm{E}-09$ & $8.90 \mathrm{E}-17$ & $1.94 \mathrm{E}-18$ \\
\hline & & & & & & Averages: & 0.14 & $3.89 \mathrm{E}-02$ & & 1.91 & $1.16 \mathrm{E}-09$ & $9.32 \mathrm{E}-17$ & \\
\hline & & & & & & 1 & 0.27 & $1.34 \mathrm{E}-02$ & \multirow[t]{4}{*}{0.40} & 1.55 & $4.78 \mathrm{E}-10$ & $3.82 \mathrm{E}-17$ & \\
\hline & & & & & & 2 & 0.27 & $1.68 \mathrm{E}-02$ & & 1.78 & $5.21 \mathrm{E}-10$ & $4.16 \mathrm{E}-17$ & $6.28 \mathrm{E}-19$ \\
\hline & & & & & & 3 & 0.27 & $2.47 \mathrm{E}-02$ & & 2.90 & $4.70 \mathrm{E}-10$ & $3.76 \mathrm{E}-17$ & $5.90 \mathrm{E}-19$ \\
\hline & & & & & & Averages: & 0.27 & $1.83 \mathrm{E}-02$ & & 2.08 & $4.90 \mathrm{E}-10$ & $3.91 \mathrm{E}-17$ & $8.63 \mathrm{E}-19$ \\
\hline & & & & & & 1 & 0.41 & $8.22 \mathrm{E}-03$ & \multirow[t]{4}{*}{0.38} & 1.55 & $2.93 \mathrm{E}-10$ & $2.34 \mathrm{E}-17$ & \\
\hline & & & & & & 2 & 0.41 & $1.08 \mathrm{E}-02$ & & 1.79 & $3.34 \mathrm{E}-10$ & $2.67 \mathrm{E}-17$ & 3.37E-19 \\
\hline & & & & & & 3 & 0.41 & $1.54 \mathrm{E}-02$ & & 2.91 & 2.92E-10 & $2.33 \mathrm{E}-17$ & $3.88 \mathrm{E}-19$ \\
\hline & & & & & & Averages: & 0.41 & $1.15 \mathrm{E}-02$ & & 2.08 & $3.06 \mathrm{E}-10$ & $2.45 \mathrm{E}-17$ & 3.31E-19 \\
\hline \multirow[t]{12}{*}{ 48R-1-WR, 28-37 } & \multirow[t]{12}{*}{359.87} & 0.056 & 0.065 & Silty clay & 46.14 & 1 & 0.14 & $8.39 \mathrm{E}-04$ & 0.45 & 1.18 & $3.20 \mathrm{E}-10$ & $2.55 \mathrm{E}-17$ & $2.85 \mathrm{E}-19$ \\
\hline & & & & & & 2 & 0.14 & $1.53 \mathrm{E}-03$ & & 2.26 & $3.03 \mathrm{E}-10$ & $2.42 \mathrm{E}-17$ & $1.70 \mathrm{E}-19$ \\
\hline & & & & & & 3 & 0.13 & $4.14 \mathrm{E}-04$ & & 0.66 & $2.81 \mathrm{E}-10$ & $2.25 \mathrm{E}-17$ & $4.58 \mathrm{E}-19$ \\
\hline & & & & & & Averages: & 0.14 & $9.27 \mathrm{E}-04$ & & & $3.01 \mathrm{E}-10$ & $2.41 \mathrm{E}-17$ & \\
\hline & & & & & & 1 & 0.34 & $3.36 \mathrm{E}-04$ & 0.43 & 0.66 & $2.28 \mathrm{E}-10$ & $1.82 \mathrm{E}-17$ & $4.44 \mathrm{E}-19$ \\
\hline & & & & & & 2 & 0.35 & $8.85 \mathrm{E}-04$ & & 2.26 & $1.76 \mathrm{E}-10$ & $1.40 \mathrm{E}-17$ & $1.89 \mathrm{E}-19$ \\
\hline & & & & & & 3 & 0.34 & $4.81 \mathrm{E}-04$ & & 1.18 & $1.83 \mathrm{E}-10$ & $1.46 \mathrm{E}-17$ & $3.23 \mathrm{E}-19$ \\
\hline & & & & & & Averages: & 0.34 & $5.67 \mathrm{E}-04$ & & & $1.96 \mathrm{E}-10$ & $1.56 \mathrm{E}-17$ & \\
\hline & & & & & & 1 & 0.55 & $2.85 \mathrm{E}-04$ & 0.41 & 0.66 & $1.93 \mathrm{E}-10$ & $1.54 \mathrm{E}-17$ & $3.73 \mathrm{E}-19$ \\
\hline & & & & & & 2 & 0.55 & $7.61 \mathrm{E}-04$ & & 2.26 & $1.51 \mathrm{E}-10$ & $1.21 \mathrm{E}-17$ & $1.26 \mathrm{E}-19$ \\
\hline & & & & & & 3 & 0.55 & 4.79E-04 & & 1.17 & $1.83 \mathrm{E}-10$ & $1.46 \mathrm{E}-17$ & $1.99 \mathrm{E}-19$ \\
\hline & & & & & & Averages: & 0.55 & $5.08 \mathrm{E}-04$ & & & $1.76 \mathrm{E}-10$ & $1.40 \mathrm{E}-17$ & \\
\hline 52R-3-WR, 78-94.5 & 381.07 & 0.058 & 0.047 & Silty clay & 40.15 & 1 & 0.14 & $2.22 \mathrm{E}-03$ & 0.38 & 2.90 & $2.29 \mathrm{E}-10$ & $1.83 \mathrm{E}-17$ & $2.68 \mathrm{E}-19$ \\
\hline & & & & & & 2 & 0.14 & $1.02 \mathrm{E}-03$ & & 1.21 & $2.52 \mathrm{E}-10$ & $2.01 \mathrm{E}-17$ & $5.84 \mathrm{E}-19$ \\
\hline & & & & & & 3 & 0.14 & $1.33 \mathrm{E}-03$ & & 2.21 & $1.80 \mathrm{E}-10$ & $1.44 \mathrm{E}-17$ & $1.28 \mathrm{E}-19$ \\
\hline & & & & & & Averages: & 0.14 & $1.52 \mathrm{E}-03$ & & & $2.20 \mathrm{E}-10$ & $1.76 \mathrm{E}-17$ & \\
\hline
\end{tabular}


Table T1 (continued). (Continued on next page.)

\begin{tabular}{|c|c|c|c|c|c|c|c|c|c|c|c|c|c|}
\hline $\begin{array}{l}\text { Core, section, } \\
\text { interval }(\mathrm{cm})\end{array}$ & $\begin{array}{l}\text { Depth } \\
\text { (mbsf) }\end{array}$ & $\begin{array}{l}\text { Core } \\
\text { diameter } \\
(\mathrm{m})\end{array}$ & $\begin{array}{l}\text { Sample } \\
\text { length } \\
\text { (m) }\end{array}$ & Lithology & $\begin{array}{l}\text { Initial } \\
\text { porosity } \\
(\%)\end{array}$ & Run & $\begin{array}{l}\text { Effective } \\
\text { stress } \\
\text { (MPa) }\end{array}$ & $\begin{array}{l}\text { Flow rate } \\
(\mathrm{mL} / \mathrm{min})\end{array}$ & $\begin{array}{l}\text { Fractional } \\
\text { porosity }\end{array}$ & $\begin{array}{l}\text { Dhead } \\
(\mathrm{m})\end{array}$ & $\begin{array}{c}K \\
(\mathrm{~m} / \mathrm{s})\end{array}$ & $\begin{array}{c}k \\
\left(m^{2}\right)\end{array}$ & $\begin{array}{c}k \\
\text { standard } \\
\text { deviation } \\
\left(\mathrm{m}^{2}\right)\end{array}$ \\
\hline & & & & & & 1 & 0.28 & $6.44 \mathrm{E}-04$ & 0.35 & 1.21 & $1.59 \mathrm{E}-10$ & $1.27 \mathrm{E}-17$ & $3.19 \mathrm{E}-19$ \\
\hline & & & & & & 2 & 0.27 & $9.89 \mathrm{E}-04$ & & 2.21 & $1.34 \mathrm{E}-10$ & 1.07E-17 & $1.02 \mathrm{E}-19$ \\
\hline & & & & & & 3 & 0.28 & $1.26 \mathrm{E}-03$ & & 2.59 & 1.46E-10 & 1.17E-17 & $8.80 \mathrm{E}-20$ \\
\hline & & & & & & Averages: & 0.28 & $9.65 \mathrm{E}-04$ & & & $1.46 \mathrm{E}-10$ & $1.17 \mathrm{E}-17$ & \\
\hline & & & & & & 1 & 0.41 & $1.18 \mathrm{E}-03$ & 0.31 & 2.90 & $1.22 \mathrm{E}-10$ & $9.74 \mathrm{E}-18$ & $1.23 \mathrm{E}-19$ \\
\hline & & & & & & 2 & 0.42 & $6.40 \mathrm{E}-04$ & & 1.90 & $1.01 \mathrm{E}-10$ & $8.05 \mathrm{E}-18$ & $8.29 \mathrm{E}-20$ \\
\hline & & & & & & 3 & 0.41 & $5.26 \mathrm{E}-04$ & & 1.53 & $1.03 \mathrm{E}-10$ & $8.25 \mathrm{E}-18$ & $6.30 \mathrm{E}-20$ \\
\hline & & & & & & Averages: & 0.41 & $7.82 \mathrm{E}-04$ & & & $1.09 \mathrm{E}-10$ & $8.68 \mathrm{E}-18$ & \\
\hline \multirow[t]{8}{*}{ 55R-1-WR, 28.5-36 } & 391.36 & 0.058 & 0.070 & Silty clay & 46.18 & 1 & 0.14 & $1.63 \mathrm{E}-03$ & 0.46 & 2.92 & $2.48 \mathrm{E}-10$ & $1.98 \mathrm{E}-17$ & $1.98 \mathrm{E}-17$ \\
\hline & & & & & & 2 & 0.14 & $5.37 \mathrm{E}-04$ & & 1.20 & $1.99 \mathrm{E}-10$ & $1.59 \mathrm{E}-17$ & $1.59 \mathrm{E}-17$ \\
\hline & & & & & & 3 & 0.14 & $1.05 \mathrm{E}-03$ & & 2.23 & $2.10 \mathrm{E}-10$ & $1.68 \mathrm{E}-17$ & $1.68 \mathrm{E}-17$ \\
\hline & & & & & & Averages: & 0.14 & $1.07 \mathrm{E}-03$ & & & $2.19 \mathrm{E}-10$ & $1.75 \mathrm{E}-17$ & \\
\hline & & & & & & 1 & 0.28 & $1.06 \mathrm{E}-03$ & 0.46 & 2.57 & $1.83 \mathrm{E}-10$ & $1.46 \mathrm{E}-17$ & $1.46 \mathrm{E}-17$ \\
\hline & & & & & & 2 & 0.28 & $6.27 \mathrm{E}-04$ & & 1.55 & $1.80 \mathrm{E}-10$ & $1.44 \mathrm{E}-17$ & $1.44 \mathrm{E}-17$ \\
\hline & & & & & & 3 & 0.28 & $7.62 \mathrm{E}-04$ & & 1.88 & $1.80 \mathrm{E}-10$ & $1.44 \mathrm{E}-17$ & $1.44 \mathrm{E}-17$ \\
\hline & & & & & & Averages: & 0.28 & $8.16 \mathrm{E}-04$ & & & $1.81 \mathrm{E}-10$ & $1.45 \mathrm{E}-17$ & \\
\hline \multirow{13}{*}{$\begin{array}{l}\text { 316-C0006E- } \\
\text { 19X-4-WR, 97-109 }\end{array}$} & & & & & & & & & & & & & \\
\hline & 119.62 & 0.059 & 0.090 & Sandy to silty clay & 43.08 & 1 & 0.14 & $2.51 \mathrm{E}-03$ & 0.40 & 1.16 & $3.38 \mathrm{E}-10$ & $2.71 \mathrm{E}-17$ & 2.40E-19 \\
\hline & & & & & & 2 & 0.14 & $4.31 \mathrm{E}-03$ & & 2.25 & $3.00 \mathrm{E}-10$ & $2.40 \mathrm{E}-17$ & $1.79 \mathrm{E}-19$ \\
\hline & & & & & & 3 & 0.14 & $5.12 \mathrm{E}-03$ & & 2.53 & 3.17E-10 & $2.53 \mathrm{E}-17$ & $1.39 \mathrm{E}-19$ \\
\hline & & & & & & Averages: & 0.14 & $3.98 \mathrm{E}-03$ & & & $3.18 \mathrm{E}-10$ & $2.55 \mathrm{E}-17$ & \\
\hline & & & & & & 1 & 0.27 & $1.38 \mathrm{E}-03$ & 0.38 & 1.16 & $1.86 \mathrm{E}-10$ & $1.49 \mathrm{E}-17$ & $2.94 \mathrm{E}-19$ \\
\hline & & & & & & 2 & 0.27 & $2.53 \mathrm{E}-03$ & & 2.25 & $1.76 \mathrm{E}-10$ & $1.40 \mathrm{E}-17$ & $2.44 \mathrm{E}-19$ \\
\hline & & & & & & 3 & 0.27 & $3.23 \mathrm{E}-03$ & & 2.94 & $1.72 \mathrm{E}-10$ & $1.38 \mathrm{E}-17$ & $1.42 \mathrm{E}-19$ \\
\hline & & & & & & Averages: & 0.27 & $2.38 \mathrm{E}-03$ & & & $1.78 \mathrm{E}-10$ & $1.42 \mathrm{E}-17$ & \\
\hline & & & & & & 1 & 0.41 & $1.29 \mathrm{E}-03$ & 0.36 & 1.56 & $1.29 \mathrm{E}-10$ & $1.03 \mathrm{E}-17$ & $1.05 \mathrm{E}-19$ \\
\hline & & & & & & 2 & 0.41 & $1.76 \mathrm{E}-03$ & & 1.85 & $1.49 \mathrm{E}-10$ & $1.19 \mathrm{E}-17$ & $1.12 \mathrm{E}-19$ \\
\hline & & & & & & 3 & 0.41 & $2.36 \mathrm{E}-03$ & & 2.53 & $1.46 \mathrm{E}-10$ & $1.17 \mathrm{E}-17$ & $7.81 \mathrm{E}-20$ \\
\hline & & & & & & Averages: & 0.41 & $1.80 \mathrm{E}-03$ & & & $1.41 \mathrm{E}-10$ & $1.13 \mathrm{E}-17$ & \\
\hline \multirow[t]{4}{*}{$34 X-3-W R, 84-94$} & 262.09 & 0.062 & 0.087 & Silty clay & 39.47 & 1 & 0.14 & $1.44 \mathrm{E}-03$ & 0.35 & 1.54 & $5.06 \mathrm{E}-10$ & 4.05E-17 & $4.98 \mathrm{E}-19$ \\
\hline & & & & & & 2 & 0.14 & $1.59 \mathrm{E}-03$ & & 1.87 & 4.62E-10 & $3.69 \mathrm{E}-17$ & $7.90 \mathrm{E}-19$ \\
\hline & & & & & & 3 & 0.14 & 2. $25 \mathrm{E}-03$ & & 2.56 & 4.77E-10 & $3.81 \mathrm{E}-17$ & $3.79 \mathrm{E}-19$ \\
\hline & & & & & & Averages: & 0.14 & $1.76 \mathrm{E}-03$ & & & $4.82 \mathrm{E}-10$ & $3.85 \mathrm{E}-17$ & \\
\hline \multirow[t]{12}{*}{$45 X-3-W R, 65-75$} & 366.27 & 0.062 & 0.084 & Silty claystone & 41.14 & 1 & 0.14 & $9.71 \mathrm{E}-04$ & 0.39 & 1.55 & $2.94 \mathrm{E}-10$ & $2.35 \mathrm{E}-17$ & $2.83 \mathrm{E}-19$ \\
\hline & & & & & & 2 & 0.14 & $1.22 \mathrm{E}-03$ & & 1.87 & $3.03 \mathrm{E}-10$ & $2.43 \mathrm{E}-17$ & $1.79 \mathrm{E}-19$ \\
\hline & & & & & & 3 & 0.14 & $1.57 \mathrm{E}-03$ & & 2.56 & $2.86 \mathrm{E}-10$ & $2.28 \mathrm{E}-17$ & $1.30 \mathrm{E}-19$ \\
\hline & & & & & & Averages: & 0.14 & $1.25 \mathrm{E}-03$ & & & $2.94 \mathrm{E}-10$ & $2.35 \mathrm{E}-17$ & \\
\hline & & & & & & 1 & 0.27 & $2.35 \mathrm{E}-04$ & 0.37 & 1.19 & $9.23 \mathrm{E}-11$ & $7.38 \mathrm{E}-18$ & $1.86 \mathrm{E}-19$ \\
\hline & & & & & & 2 & 0.27 & $4.24 \mathrm{E}-04$ & & 2.24 & 8.87E-11 & 7.09E-18 & $4.08 \mathrm{E}-20$ \\
\hline & & & & & & 3 & 0.27 & $5.70 \mathrm{E}-04$ & & 2.93 & $9.13 \mathrm{E}-11$ & $7.30 \mathrm{E}-18$ & $1.02 \mathrm{E}-19$ \\
\hline & & & & & & Averages: & 0.27 & $4.10 \mathrm{E}-04$ & & & $9.08 \mathrm{E}-11$ & $7.26 \mathrm{E}-18$ & \\
\hline & & & & & & 1 & 0.41 & $1.46 \mathrm{E}-04$ & 0.35 & 1.19 & $5.72 \mathrm{E}-11$ & 4.57E-18 & $1.55 \mathrm{E}-19$ \\
\hline & & & & & & 2 & 0.41 & $2.70 \mathrm{E}-04$ & & 2.24 & $5.63 \mathrm{E}-11$ & 4.50E-18 & $8.60 \mathrm{E}-20$ \\
\hline & & & & & & 3 & 0.41 & 3.14E-04 & & 2.93 & $5.02 \mathrm{E}-11$ & $4.01 \mathrm{E}-18$ & $5.89 \mathrm{E}-20$ \\
\hline & & & & & & Averages: & 0.41 & $2.43 \mathrm{E}-04$ & & & $5.46 \mathrm{E}-11$ & $4.36 \mathrm{E}-18$ & \\
\hline
\end{tabular}


Table T1 (continued). (Continued on next page.)

\begin{tabular}{|c|c|c|c|c|c|c|c|c|c|c|c|c|c|}
\hline $\begin{array}{l}\text { Core, section, } \\
\text { interval }(\mathrm{cm})\end{array}$ & $\begin{array}{l}\text { Depth } \\
\text { (mbsf) }\end{array}$ & $\begin{array}{l}\text { Core } \\
\text { diameter } \\
(\mathrm{m})\end{array}$ & $\begin{array}{l}\text { Sample } \\
\text { length } \\
\text { (m) }\end{array}$ & Lithology & $\begin{array}{l}\text { Initial } \\
\text { porosity } \\
\text { (\%) }\end{array}$ & Run & $\begin{array}{l}\text { Effective } \\
\text { stress } \\
(\mathrm{MPa})\end{array}$ & $\begin{array}{l}\text { Flow rate } \\
(\mathrm{mL} / \mathrm{min})\end{array}$ & $\begin{array}{l}\text { Fractional } \\
\text { porosity }\end{array}$ & $\begin{array}{c}\Delta \text { head } \\
(\mathrm{m})\end{array}$ & $\begin{array}{c}K \\
(\mathrm{~m} / \mathrm{s})\end{array}$ & $\begin{array}{c}k \\
\left(m^{2}\right)\end{array}$ & $\begin{array}{c}k \\
\text { standard } \\
\text { deviation } \\
\left(\mathrm{m}^{2}\right)\end{array}$ \\
\hline \multicolumn{14}{|l|}{ 316-C0006F- } \\
\hline \multirow[t]{4}{*}{ 17R-1-WW, 65-70* } & 542.91 & 0.025 & 0.028 & Silty claystone & 38.64 & 1 & 19.62 & $1.62 \mathrm{E}-05$ & 0.24 & 1.61 & $3.39 \mathrm{E}-12$ & $2.71 \mathrm{E}-19$ & $6.98 \mathrm{E}-20$ \\
\hline & & & & & & 2 & 19.62 & $1.69 \mathrm{E}-05$ & & 1.83 & $3.13 \mathrm{E}-12$ & $2.50 \mathrm{E}-19$ & $8.35 \mathrm{E}-20$ \\
\hline & & & & & & 3 & 0.14 & $2.56 \mathrm{E}-05$ & & 2.99 & $2.90 \mathrm{E}-12$ & $2.32 \mathrm{E}-19$ & $3.41 \mathrm{E}-20$ \\
\hline & & & & & & Averages: & 19.62 & $1.95 \mathrm{E}-05$ & & & $3.14 \mathrm{E}-12$ & $2.51 \mathrm{E}-19$ & \\
\hline \multicolumn{14}{|l|}{ 316-C0007C- } \\
\hline \multirow[t]{12}{*}{ 6X-4-WR, 57-67 } & 55.95 & 0.048 & 0.076 & Sand to clayey sand & 42.57 & 1 & 0.14 & $1.70 \mathrm{E}-03$ & 0.36 & 1.17 & $1.01 \mathrm{E}-09$ & $8.04 \mathrm{E}-17$ & 7.31E-19 \\
\hline & & & & & & 2 & 0.14 & 4.07E-03 & & 2.23 & $1.27 \mathrm{E}-09$ & $1.01 \mathrm{E}-16$ & $1.24 \mathrm{E}-18$ \\
\hline & & & & & & 3 & 0.14 & $5.11 \mathrm{E}-03$ & & 2.91 & $1.22 \mathrm{E}-09$ & $9.76 \mathrm{E}-17$ & $9.63 \mathrm{E}-19$ \\
\hline & & & & & & Averages: & 0.14 & $3.62 \mathrm{E}-03$ & & & $1.17 \mathrm{E}-09$ & $9.30 \mathrm{E}-17$ & \\
\hline & & & & & & 1 & 0.27 & $1.24 \mathrm{E}-03$ & 0.37 & 1.18 & $7.33 \mathrm{E}-10$ & $5.86 \mathrm{E}-17$ & $6.99 \mathrm{E}-19$ \\
\hline & & & & & & 2 & 0.27 & $2.50 \mathrm{E}-03$ & & 2.23 & 7.77E-10 & $6.21 \mathrm{E}-17$ & $4.80 \mathrm{E}-19$ \\
\hline & & & & & & 3 & 0.27 & $2.62 \mathrm{E}-03$ & & 2.55 & $7.13 \mathrm{E}-10$ & $5.70 \mathrm{E}-17$ & $8.33 \mathrm{E}-19$ \\
\hline & & & & & & Averages: & 0.27 & $2.12 \mathrm{E}-03$ & & & $7.41 \mathrm{E}-10$ & $5.92 \mathrm{E}-17$ & \\
\hline & & & & & & 1 & 0.41 & $1.44 \mathrm{E}-03$ & 0.31 & 1.57 & $6.39 \mathrm{E}-10$ & $5.10 \mathrm{E}-17$ & 7.96E-19 \\
\hline & & & & & & 2 & 0.41 & $2.00 \mathrm{E}-03$ & & 1.88 & $7.39 \mathrm{E}-10$ & $5.91 \mathrm{E}-17$ & 8.29E-19 \\
\hline & & & & & & 3 & 0.41 & $2.50 \mathrm{E}-03$ & & 2.94 & $6.03 \mathrm{E}-10$ & $4.82 \mathrm{E}-17$ & 4.91E-19 \\
\hline & & & & & & Averages: & 0.41 & $1.98 \mathrm{E}-03$ & & & $6.60 \mathrm{E}-10$ & $5.28 \mathrm{E}-17$ & \\
\hline \multirow[t]{12}{*}{ 11X-01-WR, 24-34 } & 100.32 & 0.057 & 0.066 & Sandy to silty clay & 34.85 & 1 & 0.14 & $6.31 \mathrm{E}-04$ & 0.30 & 1.57 & $1.75 \mathrm{E}-10$ & $1.40 \mathrm{E}-17$ & 2.53E-19 \\
\hline & & & & & & 2 & 0.14 & $6.25 \mathrm{E}-04$ & & 1.86 & $1.46 \mathrm{E}-10$ & $1.17 \mathrm{E}-17$ & 6.01E-19 \\
\hline & & & & & & 3 & 0.14 & $9.92 \mathrm{E}-04$ & & 2.55 & $1.70 \mathrm{E}-10$ & $1.36 \mathrm{E}-17$ & $1.84 \mathrm{E}-19$ \\
\hline & & & & & & Averages: & 0.14 & $7.49 \mathrm{E}-04$ & & & $1.64 \mathrm{E}-10$ & $1.31 \mathrm{E}-17$ & \\
\hline & & & & & & 1 & 0.27 & $3.49 \mathrm{E}-04$ & 0.29 & 1.57 & $9.66 \mathrm{E}-11$ & $7.73 \mathrm{E}-18$ & $2.10 \mathrm{E}-19$ \\
\hline & & & & & & 2 & 0.27 & 4.79E-04 & & 1.86 & $1.12 \mathrm{E}-10$ & $8.96 \mathrm{E}-18$ & $2.80 \mathrm{E}-19$ \\
\hline & & & & & & 3 & 0.27 & $6.48 \mathrm{E}-04$ & & 2.55 & $1.11 \mathrm{E}-10$ & $8.85 \mathrm{E}-18$ & $1.40 \mathrm{E}-19$ \\
\hline & & & & & & Averages: & 0.27 & $4.92 \mathrm{E}-04$ & & & $1.07 \mathrm{E}-10$ & $8.51 \mathrm{E}-18$ & \\
\hline & & & & & & 1 & 0.41 & $2.27 \mathrm{E}-04$ & 0.30 & 1.18 & $8.39 \mathrm{E}-11$ & $6.71 \mathrm{E}-18$ & $2.84 \mathrm{E}-19$ \\
\hline & & & & & & 2 & 0.41 & $4.40 \mathrm{E}-04$ & & 2.25 & $8.49 \mathrm{E}-11$ & $6.79 \mathrm{E}-18$ & $1.21 \mathrm{E}-19$ \\
\hline & & & & & & 3 & 0.41 & $6.19 \mathrm{E}-04$ & & 2.94 & $9.13 \mathrm{E}-11$ & $7.30 \mathrm{E}-18$ & $1.12 \mathrm{E}-19$ \\
\hline & & & & & & Averages: & 0.41 & $4.28 \mathrm{E}-04$ & & & $8.67 \mathrm{E}-11$ & $6.93 \mathrm{E}-18$ & \\
\hline \multicolumn{14}{|l|}{ 316-C0007D- } \\
\hline \multirow[t]{4}{*}{ 17R-2-WW, 24-34* } & 324.60 & 0.025 & 0.030 & Siltstone to silty claystone & 49.02 & 1 & 19.62 & $5.96 \mathrm{E}-05$ & 0.49 & 1.61 & $3.80 \mathrm{E}-11$ & $3.04 \mathrm{E}-18$ & 5.99E-20 \\
\hline & & & & & & 2 & 19.62 & $7.09 \mathrm{E}-05$ & & 1.83 & $3.98 \mathrm{E}-11$ & $3.18 \mathrm{E}-18$ & 1.70E-19 \\
\hline & & & & & & 3 & 19.62 & $1.03 \mathrm{E}-04$ & & 2.99 & $3.56 \mathrm{E}-11$ & $2.84 \mathrm{E}-18$ & $1.84 \mathrm{E}-19$ \\
\hline & & & & & & Averages: & 19.62 & $7.79 \mathrm{E}-05$ & & & $3.78 \mathrm{E}-11$ & $3.02 \mathrm{E}-18$ & \\
\hline \multirow[t]{8}{*}{ 23R-4-WR, 4-14 } & 383.89 & 0.056 & 0.095 & Silty claystone & 49.48 & 1 & 0.14 & $6.06 \mathrm{E}-04$ & 0.49 & 1.20 & $3.21 \mathrm{E}-10$ & $2.57 \mathrm{E}-17$ & $3.42 \mathrm{E}-19$ \\
\hline & & & & & & 2 & 0.14 & $6.28 \mathrm{E}-04$ & & 2.23 & $1.79 \mathrm{E}-10$ & $1.43 \mathrm{E}-17$ & $3.08 \mathrm{E}-19$ \\
\hline & & & & & & 3 & 0.14 & $7.81 \mathrm{E}-04$ & & 2.91 & $1.70 \mathrm{E}-10$ & $1.36 \mathrm{E}-17$ & $1.35 \mathrm{E}-19$ \\
\hline & & & & & & Averages: & 0.14 & $6.71 \mathrm{E}-04$ & & & $2.23 \mathrm{E}-10$ & $1.79 \mathrm{E}-17$ & \\
\hline & & & & & & 1 & 0.27 & $1.76 \mathrm{E}-04$ & 0.48 & 1.21 & $9.28 \mathrm{E}-11$ & $7.42 \mathrm{E}-18$ & $2.64 \mathrm{E}-19$ \\
\hline & & & & & & 2 & 0.27 & $2.21 \mathrm{E}-04$ & & 2.23 & $6.13 \mathrm{E}-11$ & $5.04 \mathrm{E}-18$ & $1.06 \mathrm{E}-19$ \\
\hline & & & & & & 3 & 0.27 & $2.61 \mathrm{E}-04$ & & 2.92 & $5.68 \mathrm{E}-11$ & $4.54 \mathrm{E}-18$ & $5.16 \mathrm{E}-20$ \\
\hline & & & & & & Averages: & 0.27 & $2.19 \mathrm{E}-04$ & & & $7.03 \mathrm{E}-11$ & $5.67 \mathrm{E}-18$ & \\
\hline
\end{tabular}


Table T1 (continued). (Continued on next page.)

\begin{tabular}{|c|c|c|c|c|c|c|c|c|c|c|c|c|c|}
\hline $\begin{array}{l}\text { Core, section, } \\
\text { interval }(\mathrm{cm})\end{array}$ & $\begin{array}{l}\text { Depth } \\
\text { (mbsf) }\end{array}$ & $\begin{array}{l}\text { Core } \\
\text { diameter } \\
(\mathrm{m})\end{array}$ & $\begin{array}{c}\text { Sample } \\
\text { length } \\
(\mathrm{m})\end{array}$ & Lithology & $\begin{array}{l}\text { Initial } \\
\text { porosity } \\
\text { (\%) }\end{array}$ & Run & $\begin{array}{c}\text { Effective } \\
\text { stress } \\
(\mathrm{MPa})\end{array}$ & $\begin{array}{l}\text { Flow rate } \\
(\mathrm{mL} / \mathrm{min})\end{array}$ & $\begin{array}{l}\text { Fractional } \\
\text { porosity }\end{array}$ & $\begin{array}{c}\Delta \text { head } \\
(\mathrm{m})\end{array}$ & $\begin{array}{c}K \\
(\mathrm{~m} / \mathrm{s})\end{array}$ & $\begin{array}{c}k \\
\left(m^{2}\right)\end{array}$ & $\begin{array}{c}k \\
\text { standard } \\
\text { deviation } \\
\left(\mathrm{m}^{2}\right)\end{array}$ \\
\hline & & & & & & 1 & 0.41 & $1.36 \mathrm{E}-04$ & 0.47 & 1.20 & $7.16 \mathrm{E}-11$ & $5.72 \mathrm{E}-18$ & $3.26 \mathrm{E}-19$ \\
\hline & & & & & & 2 & 0.41 & $1.61 \mathrm{E}-04$ & & 2.23 & $4.59 \mathrm{E}-11$ & $3.67 \mathrm{E}-18$ & $8.58 \mathrm{E}-20$ \\
\hline & & & & & & 3 & 0.41 & $2.01 \mathrm{E}-04$ & & 2.92 & $4.38 \mathrm{E}-11$ & $3.50 \mathrm{E}-18$ & $3.38 \mathrm{E}-20$ \\
\hline & & & & & & Averages: & 0.41 & $1.66 \mathrm{E}-04$ & & & $5.38 \mathrm{E}-11$ & $4.30 \mathrm{E}-18$ & \\
\hline \multirow[t]{4}{*}{$29 \mathrm{R}-2-\mathrm{WR}, 77-81^{*}$} & 438.62 & 0.025 & 0.035 & Silty clay & 49.02 & 1 & 0.14 & 7.17E-05 & 0.41 & 1.15 & $2.17 \mathrm{E}-11$ & $1.73 \mathrm{E}-18$ & $2.13 \mathrm{E}-19$ \\
\hline & & & & & & 2 & 0.14 & $1.79 \mathrm{E}-04$ & & 2.29 & $2.71 \mathrm{E}-11$ & $2.17 \mathrm{E}-18$ & $4.84 \mathrm{E}-20$ \\
\hline & & & & & & 3 & 0.14 & $1.89 \mathrm{E}-04$ & & 2.52 & $2.59 \mathrm{E}-11$ & 2.07E-18 & $1.49 \mathrm{E}-19$ \\
\hline & & & & & & Averages: & 0.14 & $1.46 \mathrm{E}-04$ & & & $2.49 \mathrm{E}-11$ & $1.99 \mathrm{E}-18$ & \\
\hline \multirow{13}{*}{$\begin{array}{l}\text { 316-C0008A- } \\
\text { 6H-6-WR, } 90-100\end{array}$} & & & & & & & & & & & & & \\
\hline & 50.50 & 0.063 & 0.072 & Silty clay & 53.60 & 1 & 0.14 & $1.05 \mathrm{E}-02$ & 0.51 & 1.16 & $3.51 \mathrm{E}-09$ & $2.81 \mathrm{E}-16$ & 3.19E-18 \\
\hline & & & & & & 2 & 0.14 & $1.67 \mathrm{E}-02$ & & 2.21 & 2.95E-09 & $2.36 \mathrm{E}-16$ & 2.84E- 18 \\
\hline & & & & & & 3 & 0.14 & $2.17 \mathrm{E}-02$ & & 2.51 & $3.36 \mathrm{E}-09$ & $2.69 \mathrm{E}-16$ & $3.13 \mathrm{E}-18$ \\
\hline & & & & & & Averages: & 0.14 & $1.63 \mathrm{E}-02$ & & & $3.27 \mathrm{E}-09$ & $2.62 \mathrm{E}-16$ & \\
\hline & & & & & & 1 & 0.27 & $6.93 \mathrm{E}-03$ & 0.48 & 1.15 & $2.35 \mathrm{E}-09$ & $1.88 \mathrm{E}-16$ & $3.71 \mathrm{E}-18$ \\
\hline & & & & & & 2 & 0.27 & 1.19E-02 & & 2.20 & $2.10 \mathrm{E}-09$ & $1.68 \mathrm{E}-16$ & $2.82 \mathrm{E}-18$ \\
\hline & & & & & & 3 & 0.27 & 1.47E-02 & & 2.51 & 2.27E-09 & $1.82 \mathrm{E}-16$ & $2.68 \mathrm{E}-18$ \\
\hline & & & & & & Averages: & 0.27 & $1.12 \mathrm{E}-02$ & & & $2.24 \mathrm{E}-09$ & $1.79 \mathrm{E}-16$ & \\
\hline & & & & & & 1 & 0.41 & $6.41 \mathrm{E}-03$ & 0.49 & 1.54 & $1.62 \mathrm{E}-09$ & $1.29 \mathrm{E}-16$ & $1.85 \mathrm{E}-18$ \\
\hline & & & & & & 2 & 0.41 & $1.11 \mathrm{E}-02$ & & 2.51 & $1.72 \mathrm{E}-09$ & $1.37 \mathrm{E}-16$ & $1.68 \mathrm{E}-18$ \\
\hline & & & & & & 3 & 0.41 & 1.19E-02 & & 2.89 & $1.60 \mathrm{E}-09$ & $1.28 \mathrm{E}-16$ & $2.12 \mathrm{E}-18$ \\
\hline & & & & & & Averages: & 0.41 & $9.79 \mathrm{E}-03$ & & & $1.65 \mathrm{E}-09$ & $1.31 \mathrm{E}-16$ & \\
\hline \multirow[t]{12}{*}{$12 \mathrm{H}-6-\mathrm{WR}, 88.5-98.5$} & 106.00 & 0.062 & 0.048 & Silty clay & 49.32 & 1 & 0.14 & $5.23 \mathrm{E}-03$ & 0.37 & 1.57 & $8.77 \mathrm{E}-10$ & $7.02 \mathrm{E}-17$ & 6.77E-19 \\
\hline & & & & & & 2 & 0.14 & $9.08 \mathrm{E}-03$ & & 2.51 & $9.49 \mathrm{E}-10$ & $7.59 \mathrm{E}-17$ & $6.88 \mathrm{E}-19$ \\
\hline & & & & & & 3 & 0.14 & $7.61 \mathrm{E}-03$ & & 2.24 & $8.91 \mathrm{E}-10$ & $7.12 \mathrm{E}-17$ & $1.10 \mathrm{E}-18$ \\
\hline & & & & & & Averages: & 0.14 & $7.30 \mathrm{E}-03$ & & & $9.06 \mathrm{E}-10$ & $7.24 \mathrm{E}-17$ & \\
\hline & & & & & & 1 & 0.27 & $2.89 \mathrm{E}-03$ & 0.33 & 1.14 & $6.64 \mathrm{E}-10$ & $5.31 \mathrm{E}-17$ & $1.12 \mathrm{E}-18$ \\
\hline & & & & & & 2 & 0.27 & $6.75 \mathrm{E}-03$ & & 2.93 & $6.06 \mathrm{E}-10$ & $4.84 \mathrm{E}-17$ & $3.71 \mathrm{E}-19$ \\
\hline & & & & & & 3 & 0.27 & $4.46 \mathrm{E}-03$ & & 1.83 & $6.41 \mathrm{E}-10$ & $5.12 \mathrm{E}-17$ & $5.95 \mathrm{E}-19$ \\
\hline & & & & & & Averages: & 0.27 & $4.70 \mathrm{E}-03$ & & & $6.37 \mathrm{E}-10$ & $5.09 \mathrm{E}-17$ & \\
\hline & & & & & & 1 & 0.41 & $2.66 \mathrm{E}-03$ & 0.28 & 1.57 & $4.45 \mathrm{E}-10$ & $3.56 \mathrm{E}-17$ & $7.98 \mathrm{E}-19$ \\
\hline & & & & & & 2 & 0.41 & $3.56 \mathrm{E}-03$ & & 1.83 & $5.11 \mathrm{E}-10$ & $4.08 \mathrm{E}-17$ & $4.53 \mathrm{E}-19$ \\
\hline & & & & & & 3 & 0.41 & $4.78 \mathrm{E}-03$ & & 2.51 & $4.99 \mathrm{E}-10$ & $3.99 \mathrm{E}-17$ & $3.15 \mathrm{E}-19$ \\
\hline & & & & & & Averages: & 0.41 & $3.66 \mathrm{E}-03$ & & & $4.85 \mathrm{E}-10$ & $3.88 \mathrm{E}-17$ & \\
\hline \multirow[t]{12}{*}{ 20H-4-WR, 96-105 } & 156.33 & 0.066 & 0.075 & Silty clay & 57.29 & 1 & 0.14 & $5.82 \mathrm{E}-03$ & 0.55 & 1.16 & $1.83 \mathrm{E}-09$ & $1.47 \mathrm{E}-16$ & 1.55E-18 \\
\hline & & & & & & 2 & 0.14 & $1.03 \mathrm{E}-02$ & & 2.22 & $1.71 \mathrm{E}-09$ & $1.36 \mathrm{E}-16$ & 9.91E-19 \\
\hline & & & & & & 3 & 0.14 & $1.35 \mathrm{E}-02$ & & 2.90 & $1.70 \mathrm{E}-09$ & $1.36 \mathrm{E}-16$ & $7.11 \mathrm{E}-19$ \\
\hline & & & & & & Averages: & 0.14 & $9.86 \mathrm{E}-03$ & & & $1.75 \mathrm{E}-09$ & $1.40 \mathrm{E}-16$ & \\
\hline & & & & & & 1 & 0.27 & $3.95 \mathrm{E}-03$ & 0.54 & 1.54 & $9.39 \mathrm{E}-10$ & $7.51 \mathrm{E}-17$ & $4.71 \mathrm{E}-19$ \\
\hline & & & & & & 2 & 0.27 & $5.21 \mathrm{E}-03$ & & 1.85 & $1.03 \mathrm{E}-09$ & $8.26 \mathrm{E}-17$ & $7.00 \mathrm{E}-19$ \\
\hline & & & & & & 3 & 0.27 & $6.90 \mathrm{E}-03$ & & 2.53 & $1.00 \mathrm{E}-09$ & $7.99 \mathrm{E}-17$ & 7.05E-19 \\
\hline & & & & & & Averages: & 0.27 & $5.35 \mathrm{E}-03$ & & & $9.90 \mathrm{E}-10$ & $7.92 \mathrm{E}-17$ & \\
\hline & & & & & & 1 & 0.41 & $2.30 \mathrm{E}-03$ & 0.52 & 1.17 & $7.23 \mathrm{E}-10$ & $5.78 \mathrm{E}-17$ & 5.37E-19 \\
\hline & & & & & & 2 & 0.41 & $4.63 \mathrm{E}-03$ & & 2.22 & $7.64 \mathrm{E}-10$ & $6.11 \mathrm{E}-17$ & $5.10 \mathrm{E}-19$ \\
\hline & & & & & & 3 & 0.41 & $6.00 \mathrm{E}-03$ & & 2.90 & $7.57 \mathrm{E}-10$ & $6.05 \mathrm{E}-17$ & $3.75 \mathrm{E}-19$ \\
\hline & & & & & & Averages: & 0.41 & $4.31 \mathrm{E}-03$ & & & $7.48 \mathrm{E}-10$ & $5.98 \mathrm{E}-17$ & \\
\hline
\end{tabular}


Table T1 (continued).

\begin{tabular}{|c|c|c|c|c|c|c|c|c|c|c|c|c|c|}
\hline $\begin{array}{l}\text { Core, section, } \\
\text { interval (cm) }\end{array}$ & $\begin{array}{l}\text { Depth } \\
\text { (mbsf) }\end{array}$ & $\begin{array}{l}\text { Core } \\
\text { diameter } \\
(\mathrm{m})\end{array}$ & $\begin{array}{c}\text { Sample } \\
\text { length } \\
\text { (m) }\end{array}$ & Lithology & $\begin{array}{l}\text { Initial } \\
\text { porosity } \\
(\%)\end{array}$ & Run & $\begin{array}{l}\text { Effective } \\
\text { stress } \\
(\mathrm{MPa})\end{array}$ & $\begin{array}{l}\text { Flow rate } \\
(\mathrm{mL} / \mathrm{min})\end{array}$ & $\begin{array}{c}\text { Fractional } \\
\text { porosity }\end{array}$ & $\begin{array}{c}\Delta \text { head } \\
(\mathrm{m})\end{array}$ & $\begin{array}{c}K \\
(\mathrm{~m} / \mathrm{s})\end{array}$ & $\begin{array}{c}k \\
\left(m^{2}\right)\end{array}$ & $\begin{array}{c}k \\
\text { standard } \\
\text { deviation } \\
\left(\mathrm{m}^{2}\right)\end{array}$ \\
\hline \multirow[t]{12}{*}{$24 \mathrm{H}-4-\mathrm{WR}, 84.5-94.5$} & \multirow[t]{12}{*}{191.14} & \multirow[t]{12}{*}{0.065} & \multirow[t]{12}{*}{0.085} & \multirow[t]{12}{*}{ Sandy silt to silty clay } & \multirow[t]{12}{*}{51.57} & 1 & 0.14 & $2.09 \mathrm{E}-03$ & \multirow[t]{4}{*}{0.50} & 1.18 & 7.61E-10 & $6.08 \mathrm{E}-17$ & $5.43 \mathrm{E}-19$ \\
\hline & & & & & & 2 & 0.14 & $3.15 \mathrm{E}-03$ & & 1.87 & $7.26 \mathrm{E}-10$ & $5.80 \mathrm{E}-17$ & $2.88 \mathrm{E}-19$ \\
\hline & & & & & & 3 & 0.14 & $4.80 \mathrm{E}-03$ & & 2.91 & $7.13 \mathrm{E}-10$ & $5.70 \mathrm{E}-17$ & $2.43 \mathrm{E}-19$ \\
\hline & & & & & & Averages: & 0.14 & $3.34 \mathrm{E}-03$ & & & $7.33 \mathrm{E}-10$ & $5.86 \mathrm{E}-17$ & \\
\hline & & & & & & 1 & 0.27 & $3.00 \mathrm{E}-05$ & \multirow[t]{4}{*}{0.47} & 1.18 & $4.70 \mathrm{E}-10$ & $3.75 \mathrm{E}-17$ & 4.77E-19 \\
\hline & & & & & & 2 & 0.27 & $2.49 \mathrm{E}-03$ & & 2.22 & $4.83 \mathrm{E}-10$ & $3.86 \mathrm{E}-17$ & $3.29 \mathrm{E}-19$ \\
\hline & & & & & & 3 & 0.27 & $3.19 \mathrm{E}-03$ & & 2.91 & $4.72 \mathrm{E}-10$ & $3.77 \mathrm{E}-17$ & $1.57 \mathrm{E}-19$ \\
\hline & & & & & & Averages: & 0.27 & $1.90 \mathrm{E}-03$ & & & $4.75 \mathrm{E}-10$ & $3.79 \mathrm{E}-17$ & \\
\hline & & & & & & 1 & 0.41 & $1.03 \mathrm{E}-03$ & \multirow[t]{4}{*}{0.45} & 1.18 & $3.76 \mathrm{E}-10$ & $3.01 \mathrm{E}-17$ & $4.44 \mathrm{E}-19$ \\
\hline & & & & & & 2 & 0.41 & $2.05 \mathrm{E}-03$ & & 2.22 & $3.97 \mathrm{E}-10$ & 3.17E-17 & $2.02 \mathrm{E}-19$ \\
\hline & & & & & & 3 & 0.41 & $2.62 \mathrm{E}-03$ & & 2.91 & $3.87 \mathrm{E}-10$ & $3.10 \mathrm{E}-17$ & $5.28 \mathrm{E}-19$ \\
\hline & & & & & & Averages: & 0.41 & $1.90 \mathrm{E}-03$ & & & $3.87 \mathrm{E}-10$ & $3.09 \mathrm{E}-17$ & \\
\hline \multicolumn{14}{|l|}{ 316-C0008C- } \\
\hline \multirow[t]{12}{*}{ 5H-2-WR, 19.5-29.5 } & \multirow[t]{12}{*}{34.79} & \multirow[t]{12}{*}{0.056} & \multirow[t]{12}{*}{0.088} & \multirow[t]{12}{*}{ Silty clay } & \multirow[t]{12}{*}{58.90} & 1 & 0.14 & $4.05 \mathrm{E}-03$ & \multirow[t]{4}{*}{0.56} & 1.18 & $2.02 \mathrm{E}-09$ & $1.62 \mathrm{E}-16$ & $1.54 \mathrm{E}-18$ \\
\hline & & & & & & 2 & 0.14 & $7.01 \mathrm{E}-03$ & & 2.20 & $1.88 \mathrm{E}-09$ & $1.50 \mathrm{E}-16$ & $1.82 \mathrm{E}-18$ \\
\hline & & & & & & 3 & 0.14 & $8.51 \mathrm{E}-03$ & & 2.89 & $1.74 \mathrm{E}-09$ & $1.39 \mathrm{E}-16$ & $1.24 \mathrm{E}-18$ \\
\hline & & & & & & Averages: & 0.14 & $6.52 \mathrm{E}-03$ & & & $1.88 \mathrm{E}-09$ & $1.50 \mathrm{E}-16$ & \\
\hline & & & & & & 1 & 0.27 & $2.61 \mathrm{E}-03$ & \multirow[t]{4}{*}{0.53} & 1.18 & $1.30 \mathrm{E}-09$ & $1.04 \mathrm{E}-16$ & $1.27 \mathrm{E}-18$ \\
\hline & & & & & & 2 & 0.27 & $4.09 \mathrm{E}-03$ & & 2.21 & $1.09 \mathrm{E}-09$ & $8.72 \mathrm{E}-17$ & $6.73 \mathrm{E}-19$ \\
\hline & & & & & & 3 & 0.27 & $5.14 \mathrm{E}-03$ & & 2.90 & $1.05 \mathrm{E}-09$ & 8.37E-17 & $6.52 \mathrm{E}-19$ \\
\hline & & & & & & Averages: & 0.27 & $3.94 \mathrm{E}-03$ & & & $1.15 \mathrm{E}-09$ & $9.16 \mathrm{E}-17$ & \\
\hline & & & & & & 1 & 0.41 & $2.01 \mathrm{E}-03$ & \multirow[t]{4}{*}{0.51} & 1.19 & $9.93 \mathrm{E}-10$ & $7.94 \mathrm{E}-17$ & $9.83 \mathrm{E}-19$ \\
\hline & & & & & & 2 & 0.41 & $3.17 \mathrm{E}-03$ & & 2.23 & 8.83E-10 & $6.70 \mathrm{E}-17$ & $7.16 \mathrm{E}-19$ \\
\hline & & & & & & 3 & 0.41 & $4.05 \mathrm{E}-03$ & & 2.92 & $8.19 \mathrm{E}-10$ & $6.55 \mathrm{E}-17$ & $4.91 \mathrm{E}-19$ \\
\hline & & & & & & Averages: & 0.41 & $3.07 \mathrm{E}-03$ & & & $8.98 \mathrm{E}-10$ & $7.06 \mathrm{E}-17$ & \\
\hline \multirow[t]{12}{*}{ 25X-11-WR, 96-104.5 } & \multirow[t]{12}{*}{173.96} & \multirow[t]{12}{*}{0.056} & \multirow[t]{12}{*}{0.073} & Silty clay & 49.09 & 1 & 0.14 & $1.54 \mathrm{E}-02$ & 0.48 & 1.16 & $6.57 \mathrm{E}-09$ & $5.26 \mathrm{E}-16$ & $1.28 \mathrm{E}-17$ \\
\hline & & & & & & 2 & 0.14 & $2.34 \mathrm{E}-02$ & & 2.20 & $5.28 \mathrm{E}-09$ & $4.23 \mathrm{E}-16$ & $6.18 \mathrm{E}-18$ \\
\hline & & & & & & 3 & 0.14 & $2.52 \mathrm{E}-02$ & & 2.50 & $4.98 \mathrm{E}-09$ & $3.98 \mathrm{E}-16$ & $5.03 \mathrm{E}-18$ \\
\hline & & & & & & Averages: & 0.14 & $2.13 \mathrm{E}-02$ & & & $5.61 \mathrm{E}-09$ & $4.49 \mathrm{E}-16$ & \\
\hline & & & & & & 1 & 0.28 & $4.57 \mathrm{E}-03$ & 0.37 & 1.53 & $9.96 \mathrm{E}-09$ & $7.96 \mathrm{E}-16$ & $7.80 \mathrm{E}-15$ \\
\hline & & & & & & 2 & 0.27 & $3.58 \mathrm{E}-03$ & & 1.85 & $9.56 \mathrm{E}-10$ & $7.64 \mathrm{E}-17$ & $1.43 \mathrm{E}-18$ \\
\hline & & & & & & 3 & 0.27 & $4.39 \mathrm{E}-03$ & & 2.23 & $9.73 \mathrm{E}-10$ & $7.78 \mathrm{E}-17$ & $1.48 \mathrm{E}-18$ \\
\hline & & & & & & Averages: & 0.27 & $4.18 \mathrm{E}-03$ & & & $3.96 \mathrm{E}-09$ & $3.17 \mathrm{E}-16$ & \\
\hline & & & & & & 1 & 0.41 & $3.19 \mathrm{E}-03$ & 0.35 & 1.55 & $1.02 \mathrm{E}-09$ & $8.16 \mathrm{E}-17$ & $1.63 \mathrm{E}-18$ \\
\hline & & & & & & 2 & 0.41 & $2.84 \mathrm{E}-03$ & & 1.85 & $7.58 \mathrm{E}-10$ & $6.06 \mathrm{E}-17$ & $8.25 \mathrm{E}-19$ \\
\hline & & & & & & 3 & 0.41 & 5.87E-03 & & 2.91 & 9.99E-10 & $7.98 \mathrm{E}-17$ & $7.93 \mathrm{E}-19$ \\
\hline & & & & & & Averages: & 0.41 & $3.97 \mathrm{E}-03$ & & & $9.26 \mathrm{E}-10$ & $7.40 \mathrm{E}-17$ & \\
\hline
\end{tabular}

$*=2.5 \mathrm{~cm}$ diameter samples, for which porosity change is subject to large error. 
Table T2. Results of fabric analyses by statistical calculation, Sites C0001, C0004, C0006, C0007, and C0008.

\begin{tabular}{|c|c|c|c|c|c|c|c|}
\hline \multirow[b]{2}{*}{$\begin{array}{l}\text { Hole, core, section, } \\
\text { interval }(\mathrm{cm})\end{array}$} & \multirow[b]{2}{*}{$\begin{array}{l}\text { Depth } \\
\text { CSF }(m)\end{array}$} & \multicolumn{3}{|c|}{ Section cut perpendicular to core axis } & \multicolumn{3}{|c|}{ Section cut parallel to core axis } \\
\hline & & $\begin{array}{c}\text { Grains of } \\
\text { count }\end{array}$ & $\begin{array}{c}\text { Standard } \\
\text { deviation of } \\
\text { orientation }\left({ }^{\circ}\right)\end{array}$ & $\begin{array}{l}\text { Index of } \\
\text { orientation }\end{array}$ & $\begin{array}{c}\text { Grains of } \\
\text { count }\end{array}$ & $\begin{array}{c}\text { Standard } \\
\text { deviation of } \\
\text { orientation }\left(^{\circ}\right)\end{array}$ & $\begin{array}{l}\text { Index of } \\
\text { orientation }\end{array}$ \\
\hline \multicolumn{8}{|l|}{$315-$} \\
\hline C0001E-10-3, 5 & 82.76 & 249 & 49.7 & 0.31 & 221 & 45.0 & 0.38 \\
\hline C0001F-14H-2, 35 & 208.90 & 178 & 53.0 & 0.27 & 171 & 53.9 & 0.25 \\
\hline $\mathrm{C} 0001 \mathrm{H}-13 \mathrm{R}-6,60$ & 346.76 & 354 & 37.9 & 0.48 & 301 & 50.9 & 0.30 \\
\hline $\mathrm{C} 0001 \mathrm{H}-23 \mathrm{R}-3,0$ & 430.73 & 262 & 52.2 & 0.28 & 254 & 40.7 & 0.44 \\
\hline \multicolumn{8}{|l|}{ 316- } \\
\hline C0004C-6H-7, 0 & 51.76 & 173 & 51.2 & 0.29 & 173 & 50.6 & 0.30 \\
\hline C0004D-29R-CC, 19.5 & 277.21 & 228 & 51.1 & 0.29 & 195 & 54.9 & 0.24 \\
\hline C0004D-52R-3, 78 & 381.25 & 253 & 50.8 & 0.30 & 273 & 52.4 & 0.28 \\
\hline C0004D-55R-1, 28.5 & 391.36 & 169 & 49.3 & 0.32 & 243 & 39.2 & 0.46 \\
\hline \multicolumn{8}{|l|}{$316-$} \\
\hline C0006E-5H-4, 0 & 36.16 & 208 & 53.4 & 0.26 & 212 & 51.6 & 0.29 \\
\hline C0006E-22X 223 & 146.07 & 118 & 33.3 & 0.54 & 240 & 53.8 & 0.26 \\
\hline C0006E-27X-1, 35 & 192.33 & 226 & 50.5 & 0.30 & 273 & 55.6 & 0.23 \\
\hline C0006E-34X-3, 84 & 262.09 & 221 & 50.0 & 0.31 & 217 & 46.5 & 0.36 \\
\hline C0006E-40X-8, 54 & 323.01 & 222 & 57.4 & 0.21 & 239 & 51.3 & 0.29 \\
\hline C0006E-45X-3, 65 & 366.38 & 376 & 51.5 & 0.29 & 334 & 56.2 & 0.22 \\
\hline C0006F-10R-1, 16.5 & 476.33 & 420 & 48.0 & 0.34 & 300 & 52.1 & 0.28 \\
\hline \multicolumn{8}{|l|}{$316-$} \\
\hline C0007C-6X-4, 57 & 56.07 & 153 & 51.1 & 0.29 & 295 & 54.5 & 0.25 \\
\hline C0007C-11X-1, 24 & 100.43 & 227 & 46.7 & 0.35 & 246 & 53.5 & 0.26 \\
\hline C0007D-23R-4, 4 & 383.89 & 239 & 45.9 & 0.37 & 294 & 47.0 & 0.35 \\
\hline \multicolumn{8}{|l|}{$316-$} \\
\hline C0008A-6H-6, 90 & 50.40 & 208 & 51.6 & 0.29 & 184 & 48.5 & 0.33 \\
\hline C0008A-9H-3, 29-38 & 75.41 & 188 & 48.8 & 0.33 & 148 & 52.6 & 0.27 \\
\hline C0008A-12H-6, 88.5 & 106.08 & 302 & 48.3 & 0.33 & 286 & 53.3 & 0.26 \\
\hline C0008A-20H-4, 96 & 156.34 & 188 & 47.9 & 0.34 & 155 & 44.1 & 0.39 \\
\hline C0008A-24H-4, 84.5 & 191.15 & 337 & 54.6 & 0.24 & 258 & 51.7 & 0.29 \\
\hline C0008C-5H-2, 19.5 & 34.69 & 186 & 55.3 & 0.24 & 181 & 50.1 & 0.31 \\
\hline C0008C-11H-3, 38 & 83.33 & 271 & 53.2 & 0.26 & 319 & 43.6 & 0.40 \\
\hline C0008C-25X-11, 96 & 173.96 & 177 & 49.2 & 0.32 & 196 & 54.9 & 0.25 \\
\hline
\end{tabular}

